University of Zurich

Department of Economics

Working Paper Series

ISSN 1664-7041 (print)

ISSN 1664-705X (online)

Working Paper No. 56

\title{
A Geometric Approach to Mechanism Design
}

Jacob K. Goeree and Alexey Kushnir

Revised version, June 2013 


\title{
A Geometric Approach to MECHANISM DESIGN
}

\author{
Jacob K. Goeree and Alexey Kushnir*
}

June 5, 2013

\begin{abstract}
We develop a novel geometric approach to mechanism design using an important result in convex analysis: the duality between a closed convex set and its support function. By deriving the support function for the set of feasible interim values we extend the wellknown Maskin-Riley-Matthews-Border conditions for reduced-form auctions to social choice environments. We next refine the support function to include incentive constraints using a geometric characterization of incentive compatibility. Borrowing results from majorization theory that date back to the work of Hardy, Littlewood, and Pólya (1929) we elucidate the "ironing" procedure introduced by Myerson (1981) and Mussa and Rosen (1978). The inclusion of Bayesian and dominant strategy incentive constraints result in the same support function, which establishes equivalence between these implementation concepts. Using Hotelling's lemma we next derive the optimal mechanism for any social choice problem and any linear objective, including revenue and surplus maximization. We extend the approach to include general concave objectives by providing a fixed-point condition characterizing the optimal mechanism. We generalize reduced-form implementation to environments with multi-dimensional, correlated types, non-linear utilities, and interdependent values. When value interdependencies are linear we are able to include incentive constraints into the support function and provide a condition when the second-best allocation is ex post incentive compatible.
\end{abstract}

Keywords: mechanism design, convex set, support function, duality, majorization, ironing, Hotelling's lemma, reduced-from implementation, BIC-DIC equivalence, concave objectives, interdependent values, second-best mechanisms

*ESEI Center for Market Design, University of Zürich, Blümlisalpstrasse 10, CH-8006, Zürich, Switzerland. We gratefully acknowledge financial support from the European Research Council (ERC Advanced Investigator Grant, ESEI-249433). We thank Yuanchuan Lien, Konrad Mierendorff, Roberto Serrano, Rakesh Vohra, Philip Reny, seminar participants at Cambridge University, Paris School of Economics, Ecole Polytechnique, University College London, University of Pennsylvania, SHUFE, HKUST, UTS, Humboldt University, St. Andrews University, as well as participants at the North American Econometric Society Meeting 2012, the 4th World congress of the Game Theory Society 2012, the European Econometric Society Meeting 2012, and the Asian Meetings of the Econometric Society 2012 for useful suggestions. 


\section{Introduction}

Mechanism design concerns the creation of optimal social systems by maximizing a well-defined social welfare function taking into account resource constraints and participants' incentives and hidden information. It provides a framework to address questions like "what auction format assigns goods most efficiently or yields the highest seller revenue" and "when should a public project such as building a highway be undertaken?" The difficulty in answering these questions stems from the fact that the designer typically does not possess detailed information about bidders' valuations for the goods or about voters' preferences for the public project. A welldesigned mechanism should therefore elicit participants' private information in a truthful, or incentive compatible, manner and implement the corresponding social optimum.

The constraints imposed by incentive compatibility are generally treated separately from other more basic constraints, such as resource constraints. As a result, mechanism design theory appears to have developed differently from classical approaches to consumer and producer choice theory despite some obvious parallels. For example, in producer choice theory, the firm also maximizes a well-defined objective, its profit, over a feasible production set that reflects its resource constraints. A well-known result is that a firm's optimal production plan follows by evaluating the gradient of the profit function at output and factor prices - Hotelling's lemma. One contribution of this paper is to draw a parallel between classical choice theory and mechanism design by showing how standard micro-economics tools such as Hotelling's lemma can be used to derive optimal mechanisms.

Our approach is geometric in nature and utilizes convexity of the set of feasible outcomes, which consists of a collection of probability simplices, one for each type profile. To conveniently parameterize this high-dimensional set we employ techniques from convex analysis, a subfield of mathematics that studies properties of convex sets and functions. A key result in convex analysis is the duality between a closed convex set and its support function, which is convex and homogeneous of degree one (e.g. a firm's profit function). We exploit this duality to derive, in a surprisingly simple manner, the support function of the feasible set for a general class of social choice problems.

Despite this simplicity, our geometric approach provides a powerful new perspective on reduced form implementation. Maskin and Riley (1984) first noted that with risk-averse bidders the optimal mechanism typically cannot be found by point-wise maximization but instead requires maximization over the set of interim allocation rules. This raises the issue of reduced form implementation: "which interim allocation rules are implementable, i.e. for which interim 
allocation rule does there exist an ex post allocation rule that generates it?" For the symmetric case, Matthews (1984) provided a set of inequalities and conjectured they were both necessary and sufficient, a conjecture that was subsequently proven by Border (1991).1. Our geometric approach clarifies the origin of the so called "Maskin-Riley-Matthews-Border" conditions. They are standard duality inequalities for the set of interim feasible allocations. Importantly, our approach generalizes these conditions to arbitrary social choice problems.2

We next refine the support function to include incentive constraints using a geometric characterization of incentive compatibility. Borrowing results from majorization theory due to Hardy, Littlewood, and Pólya (1929) we provide a reinterpretation of the "ironing" procedure introduced by Mussa and Rosen (1978) and Myerson (1981). We show that the support function for the set of feasible and incentive compatible outcomes is simply the support function for the feasible set, evaluated at ironed weights ${ }^{3}$

A major question in mechanism design is whether dominant strategy incentive compatibility is more stringent than Bayesian incentive compatibility. For example, does requiring dominant strategies limit a seller's revenue? A recent contribution by Manelli and Vincent (2010) shows that for the special case of single-unit, independent private-value auctions the answer is negative: Bayesian incentive compatibility and dominant strategy incentive compatibility are equivalent in this setting. Gershkov et al. (2013) extend this equivalence result to social choice environments by generalizing a theorem due to Gutmann et al. (1991).4 Here we provide a simpler proof by showing that the support functions of the sets of interim values under Bayesian and dominant-strategy incentive compatibility are identical.

Using the support function for the set of feasible and incentive compatible outcomes we derive the optimal mechanism by applying Hotelling's lemma. In particular, we determine the optimal allocation for any social choice problem and any linear objective and show that the optimal allocation can be implemented in dominant strategies. As an illustration, we determine

\footnotetext{
${ }^{1}$ Extensions to asymmetric bidders were first provided by Border (2007), see also Mierendorff (2011) and Che et al. (2010). Alternative approaches can be found in Che, Mierendorff, and Kim (2011) who study reduced-form auctions in terms of network flow problems, Hart and Reny (2011) who characterize reducedform auctions using second-order stochastic dominance, and Vohra (2011) who employs a linear-programming approach to reduced-from auctions.

${ }^{2}$ Economics papers that rely on reduced-form implementability include Armstrong (2000), Brusco and Lopomo (2002), Pai (2009), Che, Condorelli, Kim (2010), Pai and Vohra (2010, 2011, 2012), Manelli and Vincent (2010), Asker and Cantillon (2010), Belloni, Lopomo, and Wang (2010), Hörner and Samuelson (2011), Mierendorff (2011), and Miralles (2012). See Alaei et al. (2012) and Cai et al. (2012a, 2012b) for papers in computer science literature.

${ }^{3}$ Hence, the geometric approach is an alternative to Lagrangian methods that are typically used to characterize feasible and incentive compatible outcomes (see Ledyard and Palfrey, 1999, 2007).

${ }^{4}$ See Kushnir (2013) for an extension of this result to the environments with correlated types.
} 
the optimal auction for shares when bidders have decreasing marginal valuations.

We finally discuss some extensions and applications of the geometric approach. First, for general concave objectives that depend on both agent values and transfers we provide a fixedpoint condition that characterizes the optimal mechanism. Second, we further extend the reduced-form implementation to richer environments with multi-dimensional, correlated types, non-linear utilities, and interdependent values. Third, in environments with linear interdependencies we illustrate that the equivalence between Bayesian and ex post incentive compatibility breaks down. Furthermore, when the surplus maximizing allocation, i.e. first-best, is not achievable we provide a condition when the second-best allocation rule is ex post incentive compatible.

\subsection{Organization}

In Section 2 we illustrate our approach via an example of a single-unit auction with two ex ante symmetric bidders and two possible types. For this example, we demonstrate how to derive the support function for the feasible set, how to refine it to include incentive constraints, and how the optimal mechanism follows from Hoteling's lemma. Section 3 develops the approach for social choice problems, discusses its implications for reduced form implementability, establishes equivalence of Bayesian and dominant strategy implementation, and provides the optimal (dominant strategy) mechanism for any linear objective. Section 4 presents the extensions and applications. Section 5 discusses further developments and concludes. The Appendix contains most of the proofs.

\section{A Simple Auction Example}

A central result in convex analysis concerns the duality between a closed convex set $C \subset \mathbb{R}^{n}$ and its support function $\mathcal{S}^{C}: \mathbb{R}^{n} \rightarrow \mathbb{R}$, defined as

$$
\mathcal{S}^{C}(\mathbf{W})=\sup \{\mathbf{V} \cdot \mathbf{W} \mid \mathbf{V} \in C\}
$$

with $\mathbf{V} \cdot \mathbf{W}=\sum_{i=1}^{n} V_{i} W_{i}$ being the usual inner product. The support function is homogeneous of degree one, convex, and lower semi-continuous. Conversely, any function with these properties is the support function of a closed convex set, defined as the intersection of half spaces

$$
C=\left\{\mathbf{V} \in \mathbb{R}^{n} \mid \mathbf{V} \cdot \mathbf{W} \leq \mathcal{S}^{C}(\mathbf{W}) \forall \mathbf{W} \in \mathbb{R}^{n}\right\}
$$



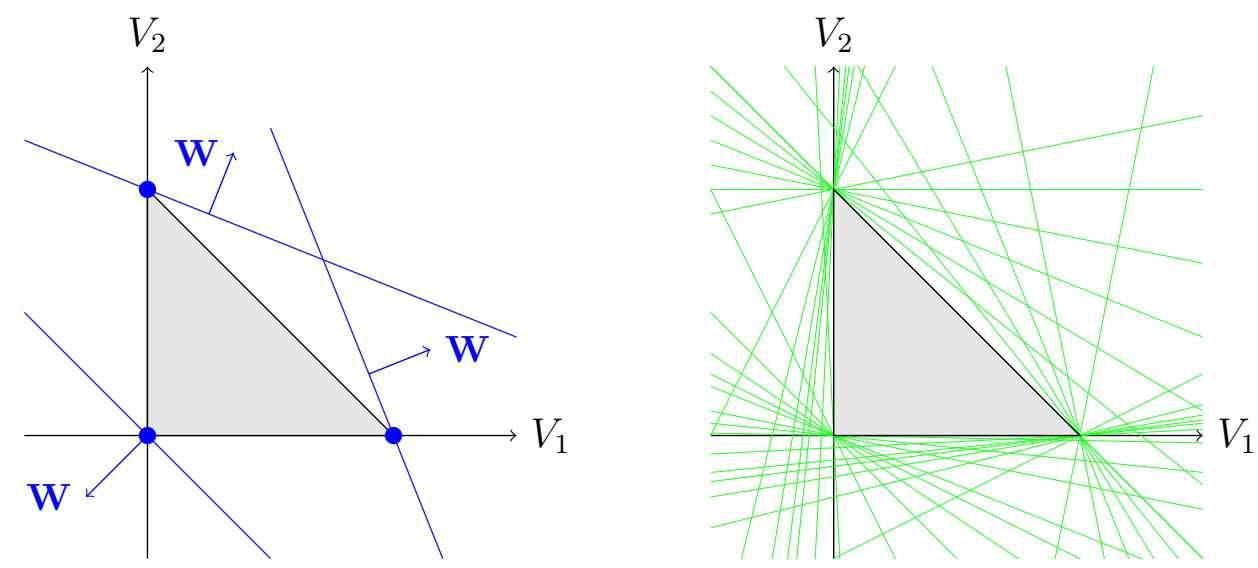

Figure 1. Illustration of duality. The left panel shows how the support function is determined by maximizing $\mathbf{V} \cdot \mathbf{W}$ over the simplex. The right panel shows how the simplex can be recovered from the inequalities $\mathbf{V} \cdot \mathbf{W} \leq \mathcal{S}(\mathbf{W})=\max \left(0, W_{1}, W_{2}\right)$ for $\mathbf{W} \in \mathbb{R}^{2}$.

Consider, for instance, the two-dimensional simplex shown in the left panel of Figure 1. In this panel, the blue dots show the outcomes of the maximization problem. It is readily verified that the support function for the simplex is

$$
\mathcal{S}(\mathbf{W})=\max \left(0, W_{1}, W_{2}\right)
$$

In turn, the simplex can be recovered from the duality inequalities $\mathbf{V} \cdot \mathbf{W} \leq \mathcal{S}(\mathbf{W})$, which define half spaces of possible $\mathbf{V}$ for each $\mathbf{W} \in \mathbb{R}^{2}$. In the right panel of Figure 1 these half spaces are bounded by the green lines and their intersection reproduces the simplex.

To illustrate our approach consider a single-unit auction with two ex ante symmetric bidders and two equally likely types, $\underline{x}<\bar{x}$. Let $\underline{Q}(\bar{Q})$ denote a bidder's expected probability of winning when her type is low (high) and define $\mathbf{Q}=(\underline{Q}, \bar{Q})$. The set of all feasible symmetric allocations is indicated by the shaded area in the left panel of Figure $25^{5}$ The support function for this set is given by:

$$
\mathcal{S}_{\text {interim }}(\mathbf{W})=\frac{1}{4} \max (0, \underline{W})+\frac{1}{2} \max (0, \underline{W}, \bar{W})+\frac{1}{4} \max (0, \bar{W})
$$

which is simply the probability-weighted sum of the support functions for the two-dimensional simplices, $\mathcal{S}(\mathbf{W})=\max \left(0, W_{1}, W_{2}\right)$, one for each of the four possible profiles.$^{6}$

\footnotetext{
${ }^{5}$ Symmetry implies that a bidder wins with probability $\frac{1}{2}$ when facing a rival of the same type, which occurs with probability $\frac{1}{2}$. Hence, the maximum expected probability of winning is $\frac{1}{2} \cdot \frac{1}{2}+\frac{1}{2} \cdot 1=\frac{3}{4}$.

${ }^{6}$ The four possible profiles for $\left(W_{1}, W_{2}\right)$ are $(\underline{W}, \underline{W}),(\underline{W}, \bar{W}),(\bar{W}, \underline{W})$, and $(\bar{W}, \bar{W})$, each of which occur with probability $\frac{1}{4}$.
} 

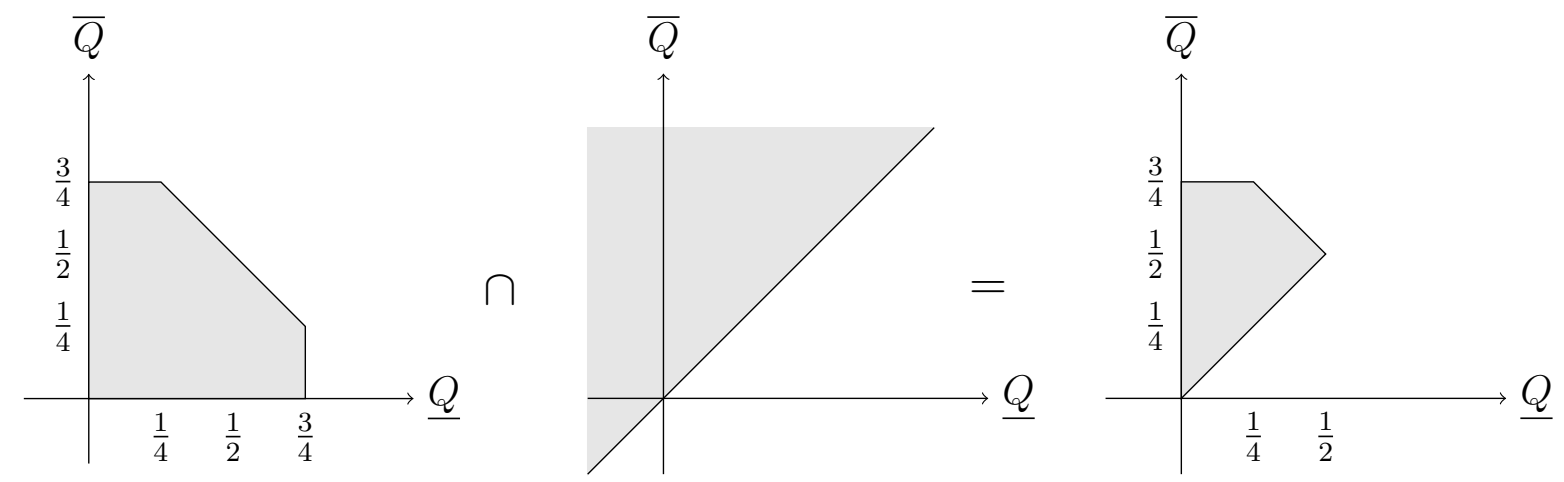

Figure 2. The figure illustrates for a single-unit auction with two-bidders and two equally-likely types that the set of feasible and Bayesian incentive compatible allocations (right panel) can be described as intersection of the set of feasible allocations (left panel) with the half-space that is "above the 45-degree line" (middle panel).

For our example, Bayesian incentive compatibility is equivalent to the monotonicity of the allocation rule, i.e. $\bar{Q} \geq \underline{Q}$ (see Section 3). To derive the support function for the set of feasible and Bayesian incentive compatible allocations it is convenient to describe this set as the intersection of the feasible set and the half-space "above the 45-degree line," see Figure 2. The half-space can be written as $(-1,1) \cdot \mathbf{Q} \geq 0$ and its support function equals

$$
\mathcal{S}^{H}(\mathbf{W})=\left\{\begin{array}{lll}
0 & \text { if } \quad \mathbf{W}=-\Lambda(-1,1) \\
\infty & \text { if } \quad \mathbf{W} \neq-\Lambda(-1,1)
\end{array}\right.
$$

for any $\Lambda \geq 0$. The support function for the intersection follows from the convolution:

$$
\begin{aligned}
\mathcal{S}_{\text {interim }}^{B I C}(\mathbf{W}) & =\inf \left\{\mathcal{S}_{\text {interim }}\left(\mathbf{W}_{1}\right)+\mathcal{S}^{H}\left(\mathbf{W}_{2}\right) \mid \mathbf{W}_{1}+\mathbf{W}_{2}=\mathbf{W}\right\} \\
& =\inf _{\Lambda \geq 0} \mathcal{S}_{\text {interim }}(\mathbf{W}+\Lambda(-1,1))
\end{aligned}
$$

It is readily verified that in optimum $\Lambda=0$ when $\underline{W} \leq \bar{W}$ and $\left.\Lambda=\frac{1}{2}(\underline{W}-\bar{W})\right)$ otherwise. To summarize, the support function for the set of feasible and Bayesian incentive-compatible allocations is simply

$$
\mathcal{S}_{\text {interim }}^{\text {BIC }}(\mathbf{W})=\mathcal{S}_{\text {interim }}\left(\mathbf{W}^{+}\right)
$$

where $\mathbf{W}^{+}$denote the "ironed" weights

$$
\mathbf{W}^{+}= \begin{cases}(\underline{W}, \bar{W}) & \text { if } \underline{W} \leq \bar{W} \\ \frac{1}{2}(\underline{W}+\bar{W}, \underline{W}+\bar{W}) & \text { if } \underline{W}>\bar{W}\end{cases}
$$




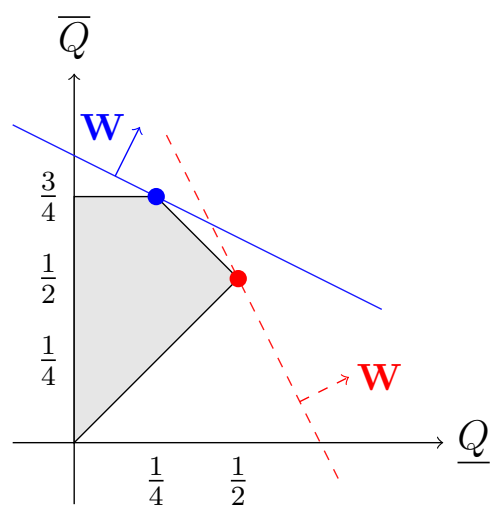

Figure 3. The figure illustrates the maximization of the linear objective over the set of feasible and Bayesian incentive compatible allocations. The lines are level-surfaces for the linear objective $\mathbf{W} \cdot \mathbf{Q}$ and the dots are optimal allocations when $0<\underline{W}<\bar{W}$ (blue) and $0<\bar{W}<\underline{W}$ (red).

We consider now the maximization of the linear objective $\mathbf{W}$. $\mathbf{Q}$ over the feasible and Bayesian incentive compatible set when the weights satisfy $0<\underline{W}<\bar{W}$, see the solid blue line on Figure 3. The support function reduces to $\mathcal{S}_{\text {interim }}\left(\mathbf{W}^{+}\right)=\frac{1}{4} \underline{W}+\frac{3}{4} \bar{W}$ and the optimal interim allocations follow from Hotelling's lemma: $\mathbf{Q}=\nabla \mathcal{S}_{\text {interim }}\left(\mathbf{W}^{+}\right)=\left(\frac{1}{4}, \frac{3}{4}\right)$ (blue dot). These expected probabilities of winning result when using an efficient symmetric allocation rule. Next suppose the weights satisfy $\underline{W}>\bar{W}>0$, see the dashed red line on Figure 3. Now the support function simplifies to $\mathcal{S}_{\text {interim }}\left(\mathbf{W}^{+}\right)=\frac{1}{2}(\underline{W}+\bar{W})$, which yields $\mathbf{Q}=\nabla \mathcal{S}_{\text {interim }}\left(\mathbf{W}^{+}\right)=\left(\frac{1}{2}, \frac{1}{2}\right)$ (red dot). This allocation results from using a random mechanism. We next generalize the above steps to social choice problems with an arbitrary number of agents and alternatives.

\section{Social Choice Implementation}

We consider an environment with a finite set $\mathcal{I}=\{1,2, \ldots, I\}$ of agents and a finite set $\mathcal{K}=\{1,2, \ldots, K\}$ of social alternatives. When alternative $k$ is selected, agent $i$ 's payoff equals $a_{i}^{k} x_{i}$ where $a_{i}^{k}$ is some constant and agent $i$ 's type, $x_{i}$, is distributed according to probability distribution $f_{i}\left(x_{i}\right)$ with discrete support $X_{i}=\left\{x_{i}^{1}, \ldots, x_{i}^{N_{i}}\right\}$, where the $x_{i}^{j}$ are non-negative with $x_{i}^{j-1}<x_{i}^{j}$ for $j=2, \ldots, N_{i} \cdot{ }^{7}$ Let $\mathbf{x}=\left(x_{1}, \ldots, x_{I}\right) \in X=\prod_{i \in \mathcal{I}} X_{i}$ denote the profile of agents' types. Without loss of generality we restrict attention to direct mechanisms characterized by

\footnotetext{
${ }^{7}$ This formulation includes many important applications, e.g. single or multi-unit auctions, public goods provision, bilateral trade, etc. For example, single-unit auctions are captured by setting $a_{i}^{k}=\delta_{i}^{k}$ for $i=1, \ldots, I$ and $k=1, \ldots, I+1$, where alternative $i=1, \ldots, I$ corresponds to the case where bidder $i$ wins the object and alternative $I+1$ corresponds to the case where the seller keeps the object. As another example, public goods provision can be summarized by two alternatives, i.e. $k=1$ when the public good is implemented and $k=2$ when it is not, and $a_{i}^{k}=\delta_{1}^{k}$ for $i=1, \ldots, I$.
} 
$K+I$ functions, $\left\{q^{k}(\mathbf{x})\right\}_{k \in \mathcal{K}}$ and $\left\{t_{i}(\mathbf{x})\right\}_{i \in \mathcal{I}}$, where $t_{i}(\mathbf{x}) \in \mathbb{R}$ is agent $i$ 's payment and $q^{k}(\mathbf{x})$ is the probability alternative $k$ is selected. We define the ex post value $v_{i}(\mathbf{x})=\sum_{k \in \mathcal{K}} a_{i}^{k} q^{k}(\mathbf{x})$ so that agent $i$ 's utility from truthful reporting, assuming others report truthfully as well, is $u_{i}(\mathbf{x})=x_{i} v_{i}(\mathbf{x})-t_{i}(\mathbf{x})$.

\subsection{Feasibility}

The probabilities with which the alternatives occur satisfy the usual feasibility conditions $q^{k}(\mathbf{x}) \geq 0$ for $k \in \mathcal{K}$ and $\sum_{k \in \mathcal{K}} q^{k}(\mathbf{x})=1$. In other words, for each type profile $\mathbf{x} \in X$, the $q^{k}(\mathbf{x})$ define a simplex in $\mathbb{R}^{K}$. The support function for the cartesian product of sets equals the sum of support functions (see Section 13 in Rockafellar, 1970), so we can simply write the support function $\mathcal{S}: \mathbb{R}^{K|X|} \rightarrow \mathbb{R}$ as a sum, over all type profiles, of simplex support functions

$$
\mathcal{S}(\mathbf{w})=\sum_{\mathbf{x} \in X} \max _{k \in \mathcal{K}} w^{k}(\mathbf{x})
$$

Let $C \in \mathbb{R}^{n}$ be a closed convex set and let $A: \mathbb{R}^{n} \rightarrow \mathbb{R}^{m}$ be a linear transformation. Recall that $A \mathbf{v} \cdot \mathbf{w}=\mathbf{v} \cdot A^{T} \mathbf{w}$ where $A^{T}$ denotes the transpose of $A$. The support function for the transformed set $A C$ is thus given by $S^{A C}(\mathbf{w})=S^{C}\left(A^{T} \mathbf{w}\right)$ for $\mathbf{w} \in \mathbb{R}^{m}$. The support function for the set of ex post values is therefore

$$
\mathcal{S}_{\text {ex post }}(\mathbf{w})=\sum_{\mathbf{x} \in X} \max _{k \in \mathcal{K}} \sum_{i \in \mathcal{I}} a_{i}^{k} w_{i}(\mathbf{x})
$$

Throughout we distinguish interim variables using capital letters: $V_{i}\left(x_{i}\right)$ denotes the interim value, $T_{i}\left(x_{i}\right)$ denotes the interim payment, and $U_{i}\left(x_{i}\right)=V_{i}\left(x_{i}\right) x_{i}-T_{i}\left(x_{i}\right)$ denotes the interim utility of agent $i \in \mathcal{I}$. Since interim values are linear transformations of ex post values, $V_{i}\left(x_{i}\right)=$ $\sum_{\mathbf{x}_{-i}} f_{-i}\left(\mathbf{x}_{-i}\right) v_{i}(\mathbf{x})$, we can once more invoke the above rule for the support function of the transformed set. To arrive at expressions that are symmetric in the probabilities we define the interim support function using the probability-weighted inner product

$$
\mathbf{V} \cdot \mathbf{W}=\sum_{i \in \mathcal{I}} \sum_{x_{i} \in X_{i}} f_{i}\left(x_{i}\right) V_{i}\left(x_{i}\right) W_{i}\left(x_{i}\right)
$$

In other words, we multiply the interim weight $W_{i}\left(x_{i}\right)$ associated with $V_{i}\left(x_{i}\right)$ by $f_{i}\left(x_{i}\right)$ so that all terms are weighted by $f(\mathbf{x})=\prod_{i} f_{i}\left(x_{i}\right)$. Using this definition of the support function we obtain the following result. 
Proposition 1. The support function for the set of feasible interim expected values is

$$
\mathcal{S}_{\text {interim }}(\mathbf{W})=E_{\mathbf{x}}\left(\max _{k \in \mathcal{K}} \sum_{i \in \mathcal{I}} a_{i}^{k} W_{i}\left(x_{i}\right)\right)
$$

and the feasible interim values $\mathbf{V}$ satisfy $\mathbf{V} \cdot \mathbf{W} \leq \mathcal{S}_{\text {interim }}(\mathbf{W})$ for all $\mathbf{W} \in \mathbb{R}^{\sum_{i}\left|X_{i}\right|}$.

It is insightful to work out the duality inequalities for single-unit private-value auctions, which fit the social choice framework as follows: alternative $k=1, \ldots, I$ corresponds to the event when bidder $i$ wins, i.e. $a_{i}^{i}=1$ and $a_{i}^{k}=0$ for $k \neq i$, and alternative $I+1$ corresponds to the event when the seller keeps the object. In this case, the reduced form value $V_{i}\left(x_{i}\right)$ is equal to a bidder $i$ 's interim expected chance of winning $Q_{i}\left(x_{i}\right)=E_{\mathbf{x}_{-i}}\left(q^{i}(\mathbf{x})\right)$ and the support function in Proposition 1 simplifies to

$$
\mathcal{S}_{\text {interim }}(\mathbf{W})=E_{\mathbf{x}}\left(\max _{i \in \mathcal{I}}\left(0, W_{i}\left(x_{i}\right)\right)\right)
$$

An exhaustive set of inequalities follow by choosing, for each $i \in \mathcal{I}$, a subset $S_{i} \subseteq X_{i}$ and setting $W_{i}\left(x_{i}\right)=1$ for $x_{i} \in S_{i}$ and 0 otherwise and then varying the set $S_{i}$.

Proposition 2. For single unit auctions, the duality inequalities simplify to

$$
\sum_{i \in \mathcal{I}} \sum_{x_{i} \in S_{i}} f_{i}\left(x_{i}\right) Q_{i}\left(x_{i}\right) \leq 1-\prod_{i \in \mathcal{I}} \sum_{x_{i} \notin S_{i}} f_{i}\left(x_{i}\right)
$$

for any subset $S_{i} \subseteq X_{i}, i=1, \ldots, I$.

The inequalities in Proposition 2 are known as the Maskin-Riley-Matthews-Border conditions for reduced form implementation, an approach to optimal auctions initiated by Maskin and Riley (1984). They were conjectured to be necessary and sufficient by Matthews (1984) based on the following intuition: the probability that a certain bidder with a certain type wins (left side) can be no higher than the probability that such a bidder exists (right side). The conjecture was subsequently proven and generalized by Border $(1991,2007)$. Besides clarifying their origin as basic duality inequalities, Proposition 1 extends these conditions to social choice problems. In Section 4 we further extend the reduced form implementation to environments with multidimensional, correlated types, non-linear utilities, and interdependent values. 


\subsection{Incentive Compatibility}

A mechanism is dominant strategy incentive compatible (DIC) if truthful reporting is a dominant strategy equilibrium 8 Necessary and sufficient conditions for a mechanism $(\mathbf{q}, \mathbf{t})$ to be DIC is that the ex post values and payments satisfy

$$
\left(v_{i}\left(x_{i}^{j}, \mathbf{x}_{-i}\right)-v_{i}\left(x_{i}^{j-1}, \mathbf{x}_{-i}\right)\right) x_{i}^{j-1} \leq t_{i}\left(x_{i}^{j}, \mathbf{x}_{-i}\right)-t_{i}\left(x_{i}^{j-1}, \mathbf{x}_{-i}\right) \leq\left(v_{i}\left(x_{i}^{j}, \mathbf{x}_{-i}\right)-v_{i}\left(x_{i}^{j-1}, \mathbf{x}_{-i}\right)\right) x_{i}^{j}
$$

for $j=2, \ldots, N_{i}$. Moreover, ex post individual rationality (EXIR) requires that $u_{i}(\mathbf{x}) \geq 0$ for $\mathbf{x} \in X, i \in \mathcal{I}$. Since agent utilities satisfy the single crossing condition the EXIR constraints are binding only for the lowest type: $t_{i}\left(x_{i}^{1}, \mathbf{x}_{-i}\right) \leq v_{i}\left(x_{i}^{1}, \mathbf{x}_{-i}\right) x_{i}^{1}$. This constraint can be included as the upward incentive constraint (3) for $j=1$ if we set $v_{i}\left(x_{i}^{0}, \mathbf{x}_{-i}\right)=t_{i}\left(x_{i}^{0}, \mathbf{x}_{-i}\right)=0$.

Similarly, a mechanism (q, t) is Bayesian incentive compatible (BIC) if truthful reporting is a Bayes-Nash equilibrium. Bayesian incentive compatibility holds if and only if the interim values and payments satisfy

$$
\left(V_{i}\left(x_{i}^{j}\right)-V_{i}\left(x_{i}^{j-1}\right)\right) x_{i}^{j-1} \leq T_{i}\left(x_{i}^{j}\right)-T_{i}\left(x_{i}^{j-1}\right) \leq\left(V_{i}\left(x_{i}^{j}\right)-V_{i}\left(x_{i}^{j-1}\right)\right) x_{i}^{j}
$$

for $j=2, \ldots, N_{i}$. Furthermore, interim individual rationality (INIR) requires that $U_{i}\left(x_{i}\right) \geq 0$ for all $x_{i} \in X_{i}, i \in \mathcal{I}$, which again holds if $T_{i}\left(x_{i}^{1}\right) \leq V_{i}\left(x_{i}^{1}\right) x_{i}^{1}$. This constraint can also be included as the upward incentive constraint for $j=1$ if we set $V_{i}\left(x_{i}^{0}\right)=T_{i}\left(x_{i}^{0}\right)=0$.

To evaluate the BIC and DIC constraints from an agent's viewpoint we determine how they affect the interim support function. In Section 2 we illustrate how to obtain the support function of the intersection of interim feasible set and the incentive constraint represented by a half space. If incentive constraints include several half spaces $\mathbf{B}_{m} \cdot \mathbf{V} \geq 0$ for $m=1, \ldots, M$ the support functions of intersection can be calculated as (see Section 13 Rockafellar, 1970)

$$
\inf _{\Lambda_{m} \geq 0} S_{\text {interim }}\left(\mathbf{W}+\sum_{m=1}^{M} \Lambda_{m} \mathbf{B}_{m}\right)
$$

The DIC constraints in (3) imply that ex post values are non-decreasing ${ }^{9}$ Furthermore, each of the $v_{i}\left(x_{i}^{j}, \mathbf{x}_{-i}\right)$ appears in two constraints: $v_{i}\left(x_{i}^{j+1}, \mathbf{x}_{-i}\right)-v_{i}\left(x_{i}^{j}, \mathbf{x}_{-i}\right) \geq 0$ and $v_{i}\left(x_{i}^{j}, \mathbf{x}_{-i}\right)-$ $v_{i}\left(x_{i}^{j-1}, \mathbf{x}_{-i}\right) \geq 0$. Let $\lambda_{i}\left(x_{i}^{j}, \mathbf{x}_{-i}\right)$ be associated with the first constraint and $\lambda_{i}\left(x_{i}^{j-1}, \mathbf{x}_{-i}\right)$ with

\footnotetext{
${ }^{8}$ More precisely, $q_{i}\left(x_{i}, \mathbf{x}_{-i}\right) x_{i}-t_{i}\left(x_{i}, \mathbf{x}_{-i}\right) \geq q_{i}\left(x_{i}^{\prime}, \mathbf{x}_{-i}\right) x_{i}-t_{i}\left(x_{i}^{\prime}, \mathbf{x}_{-i}\right)$ for all $i \in \mathcal{I}, x_{i}^{\prime}, x_{i} \in X_{i}$ and $x_{-i} \in X_{-i}$.

${ }^{9}$ Moreover, monotonicity of ex post values is also sufficient for DIC implementation: given non-decreasing ex post values one can always finds transfers that satisfy (3).
} 
the second. Likewise, the BIC constraints in (4) imply that interim values are non-decreasing and each $V_{i}\left(x_{i}^{j}\right)$ appears in two constraints, for which we similarly denote $\Lambda_{i}\left(x_{i}^{j}\right)$ and $\Lambda_{i}\left(x_{i}^{j-1}\right) 10$ Define for $j=1, \ldots, N_{i}$ the differences

$$
\begin{aligned}
\Delta \lambda_{i}\left(x_{i}^{j}, \mathbf{x}_{-i}\right) & \equiv \lambda_{i}\left(x_{i}^{j}, \mathbf{x}_{-i}\right)-\lambda_{i}\left(x_{i}^{j-1}, \mathbf{x}_{-i}\right) \\
\Delta \Lambda_{i}\left(x_{i}^{j}\right) & \equiv \Lambda_{i}\left(x_{i}^{j}\right)-\Lambda_{i}\left(x_{i}^{j-1}\right)
\end{aligned}
$$

with $\lambda_{i}\left(x_{i}^{0}, \mathbf{x}_{-i}\right)=\lambda_{i}\left(x_{i}^{N_{i}}, \mathbf{x}_{-i}\right)=0$ and $\Lambda_{i}\left(x_{i}^{0}\right)=\Lambda_{i}\left(x_{i}^{N_{i}}\right)=0$. Using the notion of the support function based on the probability-weighted inner product and formula (5) we obtain the following result.

Proposition 3. The support function for the set of feasible interim expected values that satisfy dominant strategy incentive compatibility is given by

$$
\mathcal{S}^{D I C}(\mathbf{W})=\inf _{0 \leq \lambda_{i}(\mathbf{x})} E_{\mathbf{x}}\left(\max _{k \in \mathcal{K}} \sum_{i \in \mathcal{I}} a_{i}^{k}\left(W_{i}\left(x_{i}\right)-\frac{\Delta \lambda_{i}(\mathbf{x})}{f_{i}\left(x_{i}\right)}\right)\right)
$$

Likewise, the support function for the set of feasible interim expected values that satisfy Bayesian incentive compatibility is

$$
\mathcal{S}^{B I C}(\mathbf{W})=\inf _{0 \leq \Lambda_{i}\left(x_{i}\right)} E_{\mathbf{x}}\left(\max _{k \in \mathcal{K}} \sum_{i \in \mathcal{I}} a_{i}^{k}\left(W_{i}\left(x_{i}\right)-\frac{\Delta \Lambda_{i}\left(x_{i}\right)}{f_{i}\left(x_{i}\right)}\right)\right)
$$

The minimization problem that defines the DIC support function involves more parameters, which could result in a lower support function (reflecting a smaller set). This is not the case, however, if the solutions to the minimization problems satisfy $\lambda_{i}\left(x_{i}, \mathbf{x}_{-i}\right)=\Lambda_{i}\left(x_{i}\right)$ for all $\mathbf{x}_{-i}$.

EXAMPLE 1. Consider again the single-unit auction example of Section 2 with two agents and two equally-likely types. Unlike in Section 2, however, we do not impose symmetry. We first include only the DIC constraints for agent 1 to the interim support functions ${ }^{11}$

$$
\begin{aligned}
\mathcal{S}(\mathbf{W}) & =\inf _{0 \leq \underline{\lambda}_{1}, \bar{\lambda}_{1}} \frac{1}{4} \max \left(0, \underline{W}_{1}-\underline{\lambda}_{1}, \underline{W}_{2}\right)+\frac{1}{4} \max \left(0, \bar{W}_{1}+\underline{\lambda}_{1}, \underline{W}_{2}\right) \\
& +\frac{1}{4} \max \left(0, \underline{W}_{1}-\bar{\lambda}_{1}, \bar{W}_{2}\right)+\frac{1}{4} \max \left(0, \bar{W}_{1}+\bar{\lambda}_{1}, \bar{W}_{2}\right)
\end{aligned}
$$

where $\underline{W}_{i}$ and $\bar{W}_{i}$ are weights associated with $\underline{x}$ and $\bar{x}$ for agent $i$ respectively, and $\underline{\lambda}_{1}$ and $\bar{\lambda}_{1}$ are associated with constraints $v_{1}(\bar{x}, \underline{x})-v_{1}(\underline{x}, \underline{x}) \geq 0$ and $v_{1}(\bar{x}, \bar{x})-v_{1}(\underline{x}, \bar{x}) \geq 0$ respectively.

\footnotetext{
${ }^{10}$ Monotonicity of interim values is also sufficient for BIC implementation.

${ }^{11}$ Without loss of generality we scale the $\lambda$ parameters by $\frac{1}{2}$.
} 
Surprisingly, there is always exists a solution to the minimization problem that does not depend on agent 2's weights $\underline{\lambda}_{1}=\bar{\lambda}_{1}=\max \left(0, \frac{1}{2}\left(\underline{W}_{1}-\bar{W}_{1}\right)\right)$. Similarly, if we introduce now the DIC constraints for agent 2 there is an optimal solution that does not depend on agent 1's weights $\underline{\lambda}_{2}=\bar{\lambda}_{2}=\max \left(0, \frac{1}{2}\left(\underline{W}_{2}-\bar{W}_{2}\right)\right)$. We can consider the minimization over parameters $\lambda_{1}$ and $\lambda_{2}$ sequentially only because of their geometric interpretation: each minimization corresponds to the intersection of the feasible set with the corresponding incentive constraint. For our example then the DIC minimization problem for any weights gives the same outcome as the BIC minimization problem. Therefore, the BIC and DIC support functions coincide.

To show the equivalence more generally we rely on results from majorization theory. Let $p_{1}, \ldots, p_{n}$ denote arbitrary non-negative numbers and consider two sequences $\boldsymbol{\sigma}$ and $\boldsymbol{\varsigma}$ with elements $\sigma_{j}, \varsigma_{j}$ for $j=1, \ldots, n$. We will write $\boldsymbol{\sigma} \succeq_{p} \varsigma$ if

$$
\begin{aligned}
\sum_{j=1}^{l} p_{j} \sigma_{j} \geq \sum_{j=1}^{l} p_{j} \varsigma_{j} \quad \text { for } l=1, \ldots, n-1 \\
\sum_{j=1}^{n} p_{j} \sigma_{j}=\sum_{j=1}^{n} p_{j} \varsigma_{j}
\end{aligned}
$$

The following result dates back to Hardy, Littlewood, and Polya (1929), see also Fuchs (1947).

Lemma 1. If $\boldsymbol{\sigma}, \boldsymbol{\varsigma}$ are non-decreasing sequences and $\boldsymbol{\sigma} \succeq_{p} \boldsymbol{\varsigma}$ then we say that $\boldsymbol{\sigma}$ p-majorizes $\boldsymbol{\varsigma}$ and we have

$$
\sum_{j=1}^{n} p_{j} g\left(\sigma_{j}\right) \leq \sum_{j=1}^{n} p_{j} g\left(\varsigma_{j}\right)
$$

for any continuous convex function $g: \mathbb{R} \rightarrow \mathbb{R}$.

Consider any sequence $\boldsymbol{\sigma}$, not necessarily non-decreasing, and let $\boldsymbol{\sigma}^{+}$denote the non-decreasing sequence such that (i) $\boldsymbol{\sigma} \succeq_{p} \boldsymbol{\sigma}^{+}$and (ii) any other non-decreasing sequence $\boldsymbol{\varsigma}$ that satisfies $\boldsymbol{\sigma} \succeq_{p} \boldsymbol{\varsigma}$ is $p$-majorized by $\boldsymbol{\sigma}^{+}$. The latter property motivates calling $\boldsymbol{\sigma}^{+}$the largest nondecreasing sequence that satisfies $\boldsymbol{\sigma} \succeq_{p} \boldsymbol{\varsigma}$. LemmaA1 in the Appendix establishes that sequence $\boldsymbol{\sigma}^{+}$is well defined and that $\boldsymbol{\varsigma}=\boldsymbol{\sigma}^{+}$is the solution to

$$
\min _{\boldsymbol{\sigma} \succeq_{p} \varsigma} \sum_{j=1}^{n} p_{j} g\left(\varsigma_{j}\right)
$$

where the minimization considers all possible sequences, which are not necessary non-decreasing. 
Now consider the BIC minimization problem in (7). Note that the shifted weights

$$
\widehat{W}_{i}\left(x_{i}\right)=W_{i}\left(x_{i}\right)-\frac{\Delta \Lambda_{i}\left(x_{i}\right)}{f_{i}\left(x_{i}\right)}
$$

satisfy $\mathbf{W}_{i} \succeq_{f_{i}} \widehat{\mathbf{W}}_{i}$ for all $i \in \mathcal{I} \cdot 12$ In other words, varying the $\Lambda_{i}\left(x_{i}\right)$ results in sequences $\widehat{\mathbf{W}}_{i}$ that are $f_{i}$-majorized by $\mathbf{W}_{i}$. Lemma A1 implies the $\Lambda_{i}\left(x_{i}\right)$ should be chosen such that $\widehat{\mathbf{W}}_{i}=\mathbf{W}_{i}^{+}$. Importantly, the same solution applies to the DIC minimization problem in (6).

PROPOSITION 4. The support function for the set of feasible interim expected values that satisfy BIC or DIC incentive compatibility constraints is given by

$$
\mathcal{S}^{B I C}(\mathbf{W})=\mathcal{S}^{D I C}(\mathbf{W})=\mathcal{S}_{\text {interim }}\left(\mathbf{W}^{+}\right)
$$

for any $\mathbf{W} \in \mathbb{R}^{\sum_{i}\left|X_{i}\right|}$ with $\mathcal{S}_{\text {interim }}$ given in Proposition 1.

\subsection{Optimal Mechanisms: Linear Objectives}

The mechanism that maximizes the linear objective $\mathbf{V} \cdot \boldsymbol{\Omega}$ over the set of feasible and incentive compatible interim values follows from applying Hotelling's lemma to the support function of Proposition 4 (see Section 13 Rockafellar, 1970). The BIC-DIC equivalence result of Proposition 4 ensures that the resulting mechanism can be written as a dominant strategy incentive compatible mechanism.

Proposition 5. For any social choice problem and any linear objective $\mathbf{V} \cdot \boldsymbol{\Omega}$, an optimal dominant strategy incentive compatible allocation is given by

$$
q^{k}(\mathbf{x})= \begin{cases}1 /|\mathcal{M}| & \text { if } k \in \mathcal{M} \\ 0 & \text { otherwise }\end{cases}
$$

where $\mathcal{M} \equiv \arg \max _{k \in \mathcal{K}} \sum_{i \in \mathcal{I}} a_{i}^{k} \Omega_{i}^{+}\left(x_{i}\right)$. Given $v_{i}(\mathbf{x})=\sum_{k \in \mathcal{K}} a_{i}^{k} q^{k}(\mathbf{x})$, optimal payments equal

$$
t_{i}(\mathbf{x})=x_{i} v_{i}(\mathbf{x})-\sum_{x_{i}^{j}<x_{i}}\left(x_{i}^{j+1}-x_{i}^{j}\right) v_{i}\left(x_{i}^{j}, \mathbf{x}_{-i}\right)
$$

Commonly studied objectives are expected surplus and expected revenue, both of which are linear in the interim expected values. Expected surplus $E_{\mathbf{x}}\left(\sum_{i \in \mathcal{I}} V_{i}\left(x_{i}\right) x_{i}\right)$ is readily written as

${ }^{12}$ Since $\sum_{j=1}^{l} \Delta \Lambda_{i}\left(x_{i}^{j}\right)=\Lambda_{i}\left(x_{i}^{l}\right)-\Lambda_{i}\left(x_{i}^{0}\right) \geq 0$ for $l=1, \ldots, N_{i}$ with equality for $l=N_{i}$. 
$\mathbf{V} \cdot \mathbf{x}$. Likewise, the upward binding incentive compatibility constraints (3) allow us to rewrite expected revenue $E_{\mathbf{x}}\left(\sum_{i} t_{i}(\mathbf{x})\right)$ as $\mathbf{V} \cdot \mathbf{M R}$, where the marginal revenues are defined as

$$
M R_{i}\left(x_{i}^{j}\right)=x_{i}^{j}-\left(x_{i}^{j+1}-x_{i}^{j}\right) \frac{1-F_{i}\left(x_{i}^{j}\right)}{f_{i}\left(x_{i}^{j}\right)}
$$

and $F_{i}\left(x_{i}^{j}\right)=\sum_{l=1}^{j} f_{i}\left(x_{i}^{l}\right)$. Hence, as an immediate corollary, Proposition 5 results in the efficient and the revenue maximizing mechanisms for any social choice problems.

With the next example we illustrate that the support function approach can be immediately extended to some environments with non-linear utilities and a continuous set of alternatives.

EXAMPle 2. Consider an auction for a single perfectly-divisible good among $I \geq 1$ ex ante symmetric bidders. Bidders' types are distributed according to a common probability distribution $f(\cdot)$ with support $X=\left\{x^{1}, \ldots, x^{N}\right\}$ for some $N \geq 1$. Suppose bidders have diminishing marginal valuations, i.e. they value winning a fraction $q_{i}(\mathbf{x})$ at $v_{i}(\mathbf{x})=q_{i}(\mathbf{x})^{1-\gamma}$ where $0 \leq \gamma \leq 1$. The interim support function equals

$$
\mathcal{S}_{\text {interim }}(\mathbf{W})=E_{\mathbf{x}}\left(\sum_{i=1}^{I} \max \left(0, W_{i}\left(x_{i}\right)\right)^{1 / \gamma}\right)^{\gamma}
$$

For the surplus-maximizing allocation rule we do not need to majorize agent weights and the optimal allocation equals

$$
q_{i}^{*}(\mathbf{x})=\frac{x_{i}^{1 / \gamma}}{\sum_{j=1}^{I} x_{j}^{1 / \gamma}}
$$

and the payment rule is given by

$$
t_{i}^{*}(\mathbf{x})=x_{i} q_{i}^{*}(\mathbf{x})^{1-\gamma}-\sum_{x_{i}^{j}<x_{i}}\left(x_{i}^{j+1}-x_{i}^{j}\right) q_{i}^{*}\left(x_{i}^{j}, \mathbf{x}_{-i}\right)^{1-\gamma}
$$

Similarly, the revenue-maximizing allocation rule is 13

$$
q_{i}^{* *}(\mathbf{x})=\frac{\max \left(0, M R^{+}\left(x_{i}\right)\right)^{1 / \gamma}}{\sum_{j=1}^{I} \max \left(0, M R^{+}\left(x_{j}\right)\right)^{1 / \gamma}}
$$

and the optimal payments are similarly defined ${ }^{14}$ When $\gamma$ tends to one, the efficient allocation rule assigns shares proportionally to bidders' types while the optimal allocation rule assigns

\footnotetext{
${ }^{13}$ Where we interpret $0 / 0$ as 0 .

${ }^{14}$ See Polishchuk and Tonis (2013) for results on optimal mechanisms in divisible good allocation problems.
} 
shares proportionally to bidders' marginal revenues. For intermediate values, $0<\gamma<1$, the efficient and optimal allocation rules resemble "Tullock-type" success functions. Finally, Myerson's (1981) familiar result for the optimal auction is obtained in the limit when $\gamma$ tends to zero, which corresponds to a linear value function $v_{i}(\mathbf{x})=q_{i}(\mathbf{x})$. Now the efficient allocation rule is to assign all shares to the highest-type bidder while the revenue-maximizing allocation rule assigns all shares to the bidder with the highest positive marginal revenue (and assigns nothing if all marginal revenues are negative).

\section{Extensions and Applications}

\subsection{Optimal Mechanisms: Concave Objectives}

General objectives that differ from pure profit and efficiency maximization are widespread. Many governmental programs give preferences to some groups to alleviate their economic and social disadvantages. For example, the US Federal Communications Commission gives substantial bidding preferences to minority firms in the form of bidder-subsides (Ayres and Crampton, 1996), i.e. by charging only a portion of a winning bid. In the same way, the Department of Defense charges $50 \%$ bid penalty to foreign agents in US defense contracts ${ }^{15}$ Many firms have also objectives that differ from pure profit maximization. European Civil Law countries such as France and Germany view big firms as "mini-societies" that take into account the impact of their decisions on the welfare of their employers, consumers, and suppliers (see, e.g. Tirole, 2001; and Magill, Quinzii, and Rochet, 2010) ${ }^{16}$

We analyze objectives that depend on both agent interim expected values and transfers. To include interim expected transfers $T_{i}\left(x_{i}\right)$ into the support function we denote their corresponding weights as $Z_{i}\left(x_{i}\right)$ for $x_{i} \in X_{i}, i=1, \ldots, I$. Since transfers are unrestricted the support function that corresponds to their feasible set (not yet taking into account incentive constraints) equals $E_{\mathbf{x}}\left(\delta\left(Z_{i}\left(x_{i}\right)=0, \forall x_{i}, \forall i\right)\right.$, where we use the standard definition of $\delta$-function that equals 0 if its argument is true and $+\infty$ otherwise. Combining this result with Proposition 1 we obtain the expression for the support function of feasible expected interim values and transfers

$$
\mathcal{S}_{\text {interim }}(\mathbf{W}, \mathbf{Z})=E_{\mathbf{x}}\left(\max _{k \in \mathcal{K}} \sum_{i \in \mathcal{I}} a_{i}^{k} W_{i}\left(x_{i}\right)\right)+E_{\mathbf{x}}\left(\delta\left(Z_{i}\left(x_{i}\right)=0, \forall x_{i}, \forall i\right)\right)
$$

\footnotetext{
15 "Defense Federal Acquisition Regulation Supplement," Part 225: Foreign Acquisition (2008), http://www.acq.osd.mil/.

${ }^{16}$ See Ledyard and Palfrey (1999) for a discussion about general welfare objectives in public good provision settings.
} 
Let the weights $Z_{i}\left(x_{i}\right)$ correspond to the interim expected payoffs $T_{i}\left(x_{i}\right)$ and define

$$
M R_{Z_{i}}\left(x_{i}^{j}\right)=x_{i}^{j} Z_{i}\left(x_{i}^{j}\right)-\frac{x_{i}^{j+1}-x_{i}^{j}}{f_{i}\left(x_{i}^{j}\right)} \sum_{l>j} f_{i}\left(x_{i}^{l}\right) Z_{i}\left(x_{i}^{l}\right)
$$

for $j=1, \ldots, N_{i}$ and $x_{i}^{N_{i}+1}=x_{i}^{N_{i}}$. Note that for $Z_{i}\left(x_{i}^{j}\right) \equiv 1$ the latter expression reduces to the standard formula for marginal revenues $(10)$. Parallel to Proposition 4 we now obtain the support function of feasible and incentive compatible interim expected values and transfers.

Proposition 6. The support function for the set of feasible interim expected values and transfers that satisfy BIC (DIC) constraints is given by

$$
\mathcal{S}^{D I C}(\mathbf{W}, \mathbf{Z})=\mathcal{S}^{B I C}(\mathbf{W}, \mathbf{Z})=E_{\mathbf{x}}\left(\max _{k \in \mathcal{K}} \sum_{i \in \mathcal{I}} a_{i}^{k}\left(W_{i}\left(x_{i}\right)+M R_{Z_{i}}\left(x_{i}\right)\right)^{+}\right)
$$

for any $\mathbf{W} \in \mathbb{R}^{\sum_{i}\left|X_{i}\right|}$ and $\mathbf{Z} \in \mathbb{R}_{+}^{\sum_{i}\left|X_{i}\right|}$

We now consider an increasing, concave, and differentiable objective $\oint^{18}$

$$
\mathcal{O}(\mathbf{V}, \mathbf{T})
$$

and extend the logic of Subsection 3.3 to characterize the maximimum of objective $\mathcal{O}$ over the set of feasible and incentive compatible interim expected values and transfers.

To characterize the optimal point we use that concave objectives have convex indifference curves. Therefore, the tangent line to objective $\mathcal{O}$ at the optimal point should separate the indifference curve of the objective and the feasible and incentive compatible set. Proposition 5 then implies that the optimal point belongs to the subdifferential of the support function at the vector of weights equal to the gradient of objective $\mathcal{O}$ at the optimal point (see Figure 4). As a result the following proposition follows.

Proposition 7. For any social choice problem and for any increasing, concave, and differentiable objective $\mathcal{O}(\mathbf{V}, \mathbf{T})$ the interim expected values and transfers that correspond to an optimal

\footnotetext{
${ }^{17}$ We consider ex post and interim individually rationality constraints as a part of DIC and BIC constraints respectively. See the discussion in the beginning of Subsection 3.2

${ }^{18}$ This class of objectives does not include functions that are decreasing in agent payments, like objectives increasing in agent utilities. We consider this important class of objectives in a companion paper Goeree and Kushnir (2013a).
} 


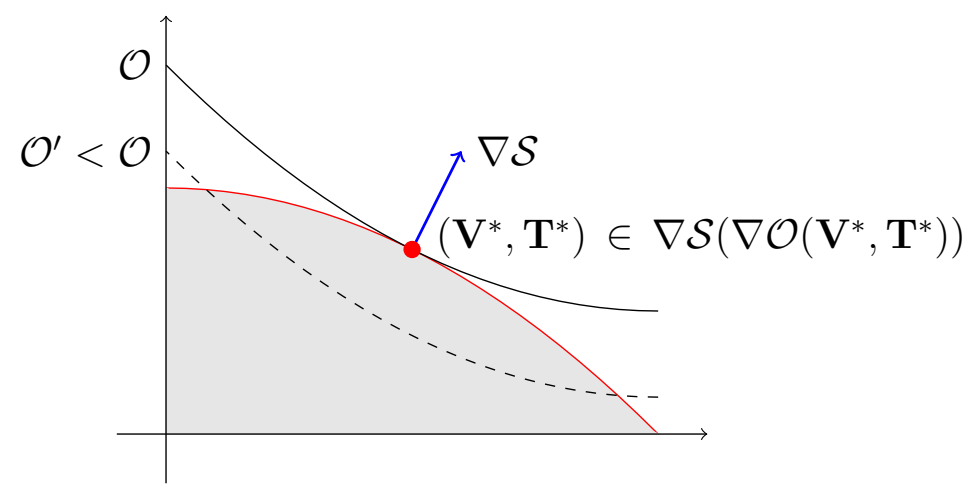

Figure 4 Maximizing concave objectives using the geometric approach. The optimal interim expected values and transfers belong to the subdifferential of the support function at the vector of weights equal to the gradient of the objective function at the optimal interim expected values and transfers.

mechanism satisfy

$$
\left(\mathbf{V}^{*}, \mathbf{T}^{*}\right) \in \nabla \mathcal{S}^{B I C}\left(\nabla \mathcal{O}\left(\mathbf{V}^{*}, \mathbf{T}^{*}\right)\right)
$$

We finally note that for increasing and concave objectives only the optimal values $\mathbf{V}^{*}$ need to be determined by the fixed-point equation (14). The optimal values $\mathbf{V}^{*}$ in turn uniquely determine the optimal payments. To understand this, we note that expression (13) links the partial derivatives of the support function with respect to $W_{i}\left(x_{i}\right)$ and the partial derivatives of the support function with respect to $Z_{i}\left(x_{i}\right)$. Using the fixed point equation (14) we then substitute the partial derivatives in this link with agent values $\mathbf{V}^{*}$ and transfers $\mathbf{T}^{*}$. As a consequence we obtain the following

$$
T_{i}^{*}\left(x_{i}\right)=V_{i}^{*}\left(x_{i}\right) x_{i}-\sum_{x_{i}^{k}<x_{i}} V_{i}^{*}\left(x_{i}^{k}\right)\left(x_{i}^{k+1}-x_{i}^{k}\right)
$$

This expression is analogous to formula (9) for the ex post optimal payments. The ex post optimal payments in (9), however, can be chosen with some flexibility. In contrast, when the objective is increasing in both agent values and transfers the optimal values determine the optimal payments uniquely. In this sense, the above expression is close to the seminal envelope theorem (e.g. Milgrom and Segal, 2002).

\footnotetext{
${ }^{19}$ We consider a differentiable objective function only for $\nabla \mathcal{O}\left(\mathbf{V}^{*}, \mathbf{T}^{*}\right)$ be a singleton. Otherwise, the optimal mechanism belongs to the subdifferential of the support function at some point in $\nabla \mathcal{O}\left(\mathbf{V}^{*}, \mathbf{T}^{*}\right)$.

${ }^{20}$ With some abuse of notation we consider the gradient $\nabla$ for the support function that takes into account the probability weighted inner product.
} 


\subsection{Reduced Form Implementation for General Social Choice Problems}

In Subsection 3.1 we show how the geometric approach extends the reduced form auctions to social choice problems for models with private values and independently distributed types. Here, we further develop this extension both to the problems with correlated and multi-dimensional types see, which is known to the literature (see Border, 2007), and to the case when agents have interdependent values, which is novel to the literature.

We consider settings with multi-dimensional types. Agent $i$ 's type is $T_{i}$-dimensional and the profile of agent types is $\mathbf{x}=\left(x_{1}^{1}, \ldots, x_{1}^{T_{1}}, \ldots, x_{I}^{1}, \ldots, x_{I}^{T_{I}}\right)$. We denote $X_{i}=\prod_{j=1}^{T_{i}} X_{i j}$, where each $X_{i j}=\left\{x_{i j}^{1}, \ldots, x_{i j}^{N_{i j}}\right\}$, and $X=\prod_{i} X_{i}$. We allow for correlation in types and denote their joint probability distribution as $f(\mathbf{x})$. Agent values are interdependent: when alternative $k$ is selected and the profile of agent types is $\mathbf{x}$ agent $i$ 's value equals $v_{i}^{k}(\mathbf{x})$. We denote agent $i$ 's ex post and interim expected value as $v_{i}(\mathbf{x})=\sum_{k \in \mathcal{K}} v_{i}^{k}(\mathbf{x}) q^{k}(\mathbf{x})$ and $V_{i}\left(x_{i}\right)=\sum_{\mathbf{x}_{-i}} f_{-i}\left(\mathbf{x}_{-i}\right) v_{i}(\mathbf{x})$ respectively.

This setup relaxes all assumptions of the main model: the values $v_{i}^{k}$ can be non-linear functions of the types, the values are not private since they depend on others' types, and types are correlated and multi-dimensional. While the setup is much more general, the derivation of the ex post and interim support functions parallels that of Proposition 1 .

Proposition 8. The support function for the feasible interim values is

$$
\mathcal{S}_{\text {interim }}(\mathbf{W})=E_{\mathbf{x}}\left(\max _{k \in \mathcal{K}} \sum_{i \in \mathcal{I}} v_{i}^{k}(\mathbf{x}) W_{i}\left(x_{i}\right)\right)
$$

and the feasible interim values $\mathbf{V}$ satisfy $\mathbf{V} \cdot \mathbf{W} \leq \mathcal{S}_{\text {interim }}(\mathbf{W})$ for all $\mathbf{W} \in \mathbb{R}^{\sum_{i}\left|X_{i}\right|}$.

Proposition 8 extends the reduced form implementation to the environments with interdependent values by providing the explicit formula for the support function for the set of agent feasible values. Yet this support function does not include incentive constraints. For the general model, determining the consequences of incentive constraints is complicated and requires more than comparing adjacent types only. We leave a complete analysis to future research but illustrate in the next subsection how our methodology applies to the case of linear interdependencies for which adjacent comparisons are sufficient. 


\subsection{Interdependent Values: Second-Best Mechanisms}

For the environments with interdependent values a natural analog of dominant strategy incentive compatibility (DIC) is the notion of ex post incentive compatibility (EPIC), which requires that, for each type profile, agents prefer to report their types truthfully when others do. EPIC and DIC coincide if agent types are private and independent (Bergemann and Moris, 2005). Unlike DIC, however, EPIC does not depend on agents' beliefs when there are value interdependencies.

In this section we illustrate how the geometric approach can be applied to environments with interdependent values. For these environments we consider settings when the maximum social surplus, i.e. first-best, is not achievable and derive its second-best level, i.e. the maximum level of social surplus under Bayesian incentive compatible (BIC) and interim individually rational (INIR) constraints. We also provide a condition when the second-best level can be implemented with EPIC and ex post individually rational (EXIR) mechanism.

Consider a single-unit auction with two bidders. Agent types are positive and independently distributed. There are only three possible social alternatives. Alternative $i$ corresponds to the event that bidder $i$ wins the unit, and alternative 0 to the event that the seller keeps the unit. The utility of agent $i$ equals $x_{i}+\alpha x_{j}$ if he wins the object and 0 otherwise. Hence, the expected interim value of agent $i$ is

$$
V_{i}\left(x_{i}\right)=x_{i} Q_{i}\left(x_{i}\right)+\alpha E_{x_{j}}\left(x_{j} q^{i}\left(x_{i}, x_{j}\right)\right),
$$

where $Q_{i}\left(x_{i}\right)=E_{x_{j}}\left(q^{i}\left(x_{i}, x_{j}\right)\right)$ is bidder $i$ 's interim expected chance of winning. In this environment, the incentive compatibility constraints reduce to monotonicity constraints on the allocation rule.

Proposition 9. If mechanism (q, $\mathbf{t})$ is BIC (EPIC) then $Q_{i}\left(x_{i}\right)\left(q^{i}\left(x_{i}, \mathbf{x}_{-i}\right)\right)$ is non-decreasing function of $x_{i}$ for each $i \in \mathcal{I}$. Conversely, for any allocation rule $\mathbf{q}$ such that $Q_{i}\left(x_{i}\right)\left(q^{i}\left(x_{i}, \mathbf{x}_{-i}\right)\right)$ is non-decreasing function of $x_{i}$ for each $i \in \mathcal{I}$ there exist transfers $\mathbf{t}$ such that mechanism $(\mathbf{q}, \mathbf{t})$ is $B I C$ (EPIC).

Given the above monotonicity conditions the support function for the set of interim expected values that can be achieved with some BIC and INIR mechanism equals

$$
S^{B I C}(\mathbf{W})=\inf _{\Lambda_{i}\left(x_{i}\right) \geq 0} E_{\mathbf{x}} \max _{i \neq j}\left(0,\left(x_{i}+\alpha x_{j}\right) W_{i}\left(x_{i}\right)-\frac{\Delta \Lambda_{i}\left(x_{i}\right)}{f_{i}\left(x_{i}\right)}\right)
$$

Similarly the support function for the set of interim expected values that can be achieved with 
some EPIC and EXIR mechanism equals

$$
S^{E P I C}(\mathbf{W})=\inf _{\lambda_{i}(\mathbf{x}) \geq 0} E_{\mathbf{x}} \max _{i \neq j}\left(0,\left(x_{i}+\alpha x_{j}\right) W_{i}\left(x_{i}\right)-\frac{\Delta \lambda_{i}(\mathbf{x})}{f_{i}\left(x_{i}\right)}\right)
$$

Maskin (1992) show for a continuous-type version of this example that the first-best social surplus cannot be implemented with BIC and INIR (and, hence, EPIC and EXIR) mechanism when $\alpha>1$. We now provide conditions when the second-best level of social surplus, which equals the value of support function at unit weights $S^{B I C}(\mathbf{1})$, can be implemented with some EPIC and EXIR mechanism. We first approach this question when the unit has to be always allocated to the agents(see also Proposition 2 in Hernando-Veciana and Michelucci, 2011), and, hence, there are no zeros in the support function expressions.

Proposition 10. If the unit has to be always allocated to the agents and $\alpha>1$ the second-best level of social surplus can be implemented with some EPIC and EXIR mechanism and equals

$$
S^{E P I C}(\mathbf{1})=S^{B I C}(\mathbf{1})=\max _{i \in \mathcal{I}}\left(E_{i}\left(x_{i}\right)\right)+\alpha \min _{i \in \mathcal{I}}\left(E_{i}\left(x_{i}\right)\right)
$$

We now identify a condition on $\alpha$ when one of the bidders always get the unit at the second-best allocation, even though the auctioneer can keep the unit (see also Proposition 6 in HernandoVeciana and Michelucci, 2009). In this case the second-best level of social surplus can be again implemented with some EPIC and EXIR mechanism.

Proposition 11. Consider the case when the auctioneer can keep the unit. The second-best level of social surplus can be implemented with some EPIC and EXIR mechanism if

$$
1<\alpha \leq \max _{i \in \mathcal{I}}\left(\frac{E_{i}\left(x_{i}\right)}{E_{i}\left(x_{i}\right)-\sum_{j \in \mathcal{I}} x_{j}^{1}}\right)
$$

where $E_{i}\left(x_{i}\right)$ denotes the expected type of agent $i$.

We finally illustrate our results with a simple example of a single-unit auction with two symmetric bidders and two equally likely and independent types, $\underline{x}=1$ and $\bar{x}=10$. We compare the sets of feasible outcomes that satisfy BIC and EPIC constraints respectively. Since the bidders are ex ante symmetric, the allocation rule has no agent specific subscript and can be represented by a matrix

$$
\mathbf{q}=\left(\begin{array}{ll}
q(\underline{x}, \underline{x}) & q(\bar{x}, \underline{x}) \\
q(\underline{x}, \bar{x}) & q(\bar{x}, \bar{x})
\end{array}\right)
$$



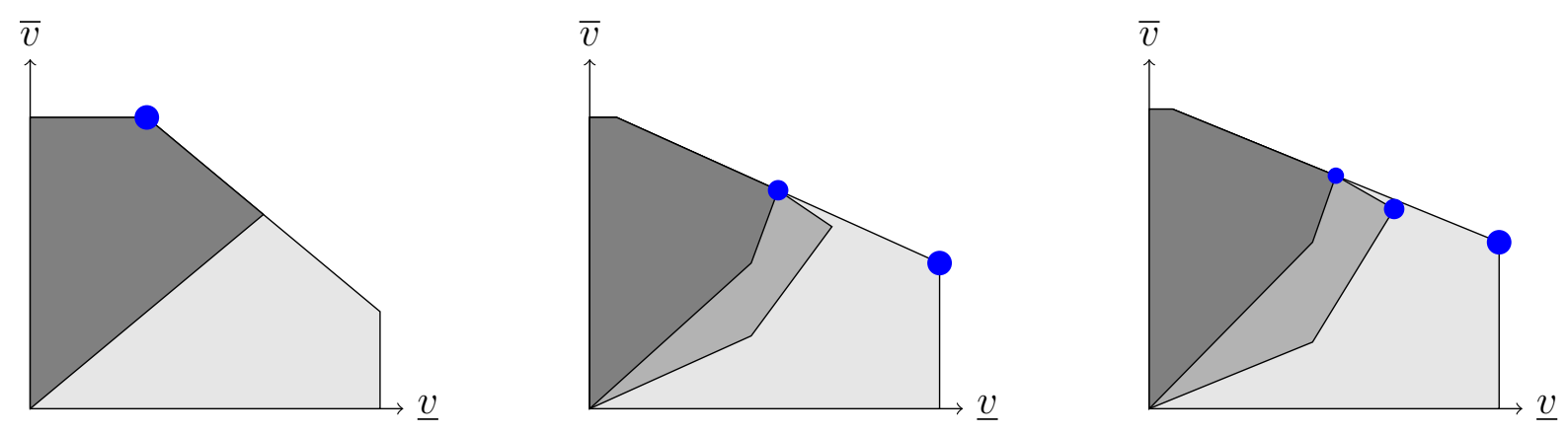

Figure 5. Application of the geometric approach to the environments with interdependent values. Shown are the feasible outcomes with no incentive constraints imposed (light), Bayesian incentive compatible outcomes (medium dark), and ex post incentive compatible outcomes (dark) for $\alpha=0$ (left panel), $\alpha=1.2$ (middle panel), and $\alpha=2$ (right panel). The largest blue dot indicates the first-best outcome, the medium-sized blue dot the second-best outcome under BIC, and the smallest blue dot indicates the allocation that delivers the maximum level of social surplus under EPIC.

where the rows correspond to (say) agent 1's type and the columns to agent 2's type, and the entries correspond to the probabilities that the object is assigned to agent 1 . The probability that object is assigned to agent 2 can be obtained by the transposition of the matrix.

Figure 5 shows the sets of interim values that result when $\alpha=0$ (left panel), $\alpha=1.2$ (middle panel), and $\alpha=2$ (right panel). In each of the panels, the light area corresponds to the set of feasible values without any incentive constraints imposed, the medium dark area to the BIC values, and the dark area to the EPIC values. In case of pure private values, i.e. $\alpha=0$, the latter two sets coincide as shown by the left panel. However, the equivalence between Bayesian and ex post implementation generally fails when $\alpha>0$ as shown by the middle and right panels.

The easiest way to describe the different sets is by their vertices ${ }^{21}$ For instance, the set of EPIC outcomes can be described by five vertices, which (clockwise starting at the origin) correspond to the following allocation rules

$$
\mathbf{q}^{E P I C}=\left(\begin{array}{ll}
0 & 0 \\
0 & 0
\end{array}\right),\left(\begin{array}{cc}
0 & 0 \\
1 & \frac{1}{2}
\end{array}\right),\left(\begin{array}{cc}
\frac{1}{2} & 0 \\
1 & \frac{1}{2}
\end{array}\right),\left(\begin{array}{cc}
\frac{1}{2} & \frac{1}{2} \\
\frac{1}{2} & \frac{1}{2}
\end{array}\right),\left(\begin{array}{ll}
0 & \frac{1}{2} \\
0 & \frac{1}{2}
\end{array}\right)
$$

Likewise, for the BIC outcomes the six vertices correspond to the allocation rules

$$
\mathbf{q}^{B I C}=\left(\begin{array}{ll}
0 & 0 \\
0 & 0
\end{array}\right),\left(\begin{array}{cc}
0 & 0 \\
1 & \frac{1}{2}
\end{array}\right),\left(\begin{array}{cc}
\frac{1}{2} & 0 \\
1 & \frac{1}{2}
\end{array}\right),\left(\begin{array}{cc}
\frac{1}{2} & \frac{1}{2} \\
\frac{1}{2} & \frac{1}{2}
\end{array}\right),\left(\begin{array}{cc}
0 & \frac{3}{4} \\
\frac{1}{4} & \frac{1}{2}
\end{array}\right),\left(\begin{array}{cc}
0 & \frac{1}{2} \\
\frac{1}{2} & 0
\end{array}\right)
$$

${ }^{21}$ The vertices follow from the gradient of the support function at points of differentiability. The five EPIC vertices $(0,0),\left(0, \frac{15}{2}+3 \alpha\right),\left(\frac{1}{4}+\frac{1}{4} \alpha, \frac{15}{2}+3 \alpha\right),\left(\frac{1}{2}+\frac{11}{4} \alpha, 5+\frac{11}{4} \alpha\right),\left(\frac{1}{4}+\frac{5}{2} \alpha, \frac{5}{2}+\frac{5}{2} \alpha\right)$. The first four plus $\left(\frac{3}{8}+\right.$ $\left.\frac{15}{4} \alpha, \frac{15}{4}+\frac{21}{8} \alpha\right),\left(\frac{1}{4}+\frac{5}{2} \alpha, \frac{5}{2}+\frac{1}{4} \alpha\right)$ constitute the six BIC vertices. 
Bayesian incentive compatibility requires that the sum of entries in the top row does not exceed the sum of entries in the bottom row. In contrast, ex post incentive compatibility requires that the entries in the top row do not exceed the entries in the bottom row for both columns (see Proposition 9). Notice that the final two BIC matrices violate this more stringent condition.

The blue dots in Figure 5 indicate the optimal outcomes: the largest blue dot indicates the first-best outcome, the medium-sized blue dot indicates the second-best outcome under $\mathrm{BIC}$, and the smallest blue dot indicates the allocation that delivers the maximum level of social surplus under EPIC. For $\alpha \leq 1$, the first-best outcome can be implemented with EPIC

mechanism and correspond to the third EPIC matrix. When $1<\alpha \leq \frac{E(x)}{E(x)-2 \underline{x}}=\frac{11}{7}$ the first-best cannot be achieved, but the second-best outcome can be implemented with EPIC mechanisms and correspond to the penultimate EPIC matrix. If $\alpha>\frac{11}{7}$ the second-best outcome can be implemented only with BIC mechanism, and correspond to the penultimate BIC matrix. In this case BIC implementation leads to more social surplus than EPIC.

\section{Conclusions}

This paper develops a novel geometric approach to mechanism design based on the duality between a convex closed set and its support function. Using this duality, we first extend the literature on reduced form auctions to social choice problems with interdependent values. Second, we show that the "ironing" procedure (Mussa and Rosen, 1978; and Myerson, 1981) can be reinterpreted in terms of majorization theory (Hardy, Littlewood, and Pólya, 1929). We also provide a simple proof that the feasible sets of interim expected values and transfers that remain after imposing Bayesian and dominant strategy incentive compatibility constraints coincide (see Gershkov et. al, 2013). Third, the knowledge of the support function admits the direct derivation of the optimal mechanism using standard tools such as Hotelling's lemma. We determine the optimal mechanism for any social choice problem and any linear objective, including revenue and surplus maximization. We also provide a fixed point equation that characterizes an optimal mechanism for general concave objectives. Finally, for environments with linear value interdependencies we provide a condition when the second-best allocation is ex post incentive compatible.

Importantly, our geometric approach applies to questions that go beyond the scope of this paper. The geometric interpretation of the incentive compatibility constraints extend to other types of constraints, such as capacity constraints and budget balance. For instance, we consider the maximization of general concave welfare objectives when agent transfers needs to be budget 
balanced in a companion paper Goeree and Kushnir (2013a). Next, the geometric approach can be immediately adapted to continuous type spaces. An analog of support functions for infinite-dimensional type-spaces are support functionals. They possess the same properties as support functions with respect to additions, intersections, and linear transformations, as well as there is an analog of Hotelling's lemma for continuous type-spaces (see Chapter 7.10 in Aliprantis and Border, 2006). Moreover, the geometric approach can be readily applied to the environments with multi-dimensional types and non-linear utilities. For these environments the incentive compatibility constraints are equivalent to cycle-monotonicity conditions (Rochet, 1987), which can be incorporated into the support function (Goeree and Kushnir, 2013b).

To summarize, our geometric approach applies to a wide spectrum economic problems. As such it may provide a powerful tool to study mechanism design problems that have hitherto resisted thorough analysis because of analytical intractability. We leave this exciting prospect as a topic for future research. 


\section{A. Appendix}

Proof of Proposition 2. Necessity of the inequalities follows from the definition of the support function. Sufficiency also follows easily from our approach by interpreting (2) in terms of hyperplanes that bound the interim expected probability set. Any boundary point of the interim expected probability set, i.e. any $\mathbf{Q}$ that satisfies $\mathbf{Q} \cdot \mathbf{W}=\mathcal{S}(\mathbf{W})$ for some $\mathbf{W}$, can be written as $\mathbf{Q}=\nabla \mathcal{S}(\mathbf{W})$ at points of differentiability of the support function from the envelope theorem ${ }^{22}$ Furthermore, if $\mathcal{S}(\mathbf{W})$ is not differentiable at $\mathbf{W}$ then the subdifferential $\nabla \mathcal{S}(\mathbf{W})$ produces a face on the boundary. For any $\mathbf{Q} \neq \mathbf{Q}^{\prime}$ that belong to such a face we have

$$
\left(\mathbf{Q}-\mathbf{Q}^{\prime}\right) \cdot \mathbf{W}=\mathcal{S}(\mathbf{W})-\mathcal{S}(\mathbf{W})=0 .
$$

Each point of non-differentiability, W, therefore defines a normal vector to the face of the polyhedron, formed by $\nabla \mathcal{S}(\mathbf{W})$. For the support function (1) the points of non-differentiability are weight vectors with several equal entries, and those equal entries are the largest entries for some profile of types $\mathbf{x}$. Since non-maximum entries does not change the value of the support function we can consider only such weights where these entries are 0 . Since the support function is homogeneous of degree one we can restrict ourselves to weights with only 1 and 0 entries. Then considering all non-trivial $\mathbf{W} \in\{0,1\}^{\sum_{i} X_{i}}$ exhausts all hyperplanes that contain one of the faces of the boundary of the interim expected probability set.

Lemma A1. For any $\boldsymbol{\sigma}$ sequence $\boldsymbol{\sigma}^{+}$is well defined and $\boldsymbol{\varsigma}=\boldsymbol{\sigma}^{+}$solves

$$
\min _{\boldsymbol{\sigma} \succeq_{p} \varsigma} \sum_{j=1}^{n} p_{j} g\left(\varsigma_{j}\right)
$$

for any convex function $g: \mathbb{R} \rightarrow \mathbb{R}$.

Proof: We first show that sequence $\boldsymbol{\sigma}^{+}$is well defined. For this purpose let us define for any non-decreasing sequence $\boldsymbol{\varsigma} \in \mathbb{R}^{n}$ function $f_{l}(\boldsymbol{\varsigma})=\sum_{j=1}^{l} p_{j} \varsigma_{j}$ and $\alpha_{l}=\sup _{\boldsymbol{\sigma} \succeq_{p} \varsigma} f_{l}(\boldsymbol{\varsigma}), l=1, . ., n$, where the supremum is taken only over non-decreasing sequences. Define now sequence $\boldsymbol{\sigma}^{+}$ as $\sigma_{l}^{+}=\left(\alpha_{l}-\alpha_{l-1}\right) / p_{l}$, where $\alpha_{0}=0$. Clearly, we have $\boldsymbol{\sigma} \succeq_{p} \boldsymbol{\sigma}^{+}$. To prove that $\boldsymbol{\sigma}^{+}$is non-decreasing notice that

$$
\frac{f_{l}(\mathbf{\varsigma})}{p_{l}}+\frac{f_{l-2}(\mathbf{\varsigma})}{p_{l-1}} \geq\left(\frac{1}{p_{l}}+\frac{1}{p_{l-1}}\right) f_{l-1}(\boldsymbol{\varsigma})
$$

for any non-decreasing $\varsigma$ and $l=2, \ldots, n$. Therefore,

$$
\sup _{\boldsymbol{\sigma} \succeq_{p} \boldsymbol{\varsigma}}\left(\frac{f_{l}(\boldsymbol{\varsigma})}{p_{l}}+\frac{f_{l-2}(\boldsymbol{\varsigma})}{p_{l-1}}\right) \geq\left(\frac{1}{p_{l}}+\frac{1}{p_{l-1}}\right) \sup _{\boldsymbol{\sigma} \succeq_{p} \boldsymbol{\varsigma}} f_{l-1}(\boldsymbol{\varsigma})
$$

\footnotetext{
${ }^{22}$ We consider the gradient $\nabla$ that takes into account the probability weighted inner product.
} 
where the supremums are taken over only non-decreasing sequences. Hence, $\left(\alpha_{l}-\alpha_{l-1}\right) / p_{l} \geq$ $\left(\alpha_{l-1}-\alpha_{l-2}\right) / p_{l-1}$.

We now consider minimization problem A.1). We show that, without loss of generality, we can restrict attention to non-decreasing sequences $\varsigma$. Suppose not and $\varsigma_{l}>\varsigma_{k}$ for $l<k$. Then define the sequence $\tilde{\boldsymbol{\varsigma}}$ with elements $\tilde{\varsigma}_{l}=\varsigma_{l}-\varepsilon\left(\varsigma_{l}-\varsigma_{k}\right) / p_{l}$ and $\tilde{\varsigma}_{k}=\varsigma_{k}+\varepsilon\left(\varsigma_{l}-\varsigma_{k}\right) / p_{k}$ while $\tilde{\varsigma}_{j}=\varsigma_{j}$ for $j \neq l, k$. The sequence $\tilde{\boldsymbol{\varsigma}}$ also satisfies $\boldsymbol{\sigma} \succeq_{p} \tilde{\boldsymbol{\varsigma}}$. Since $g(\cdot)$ is convex we have

$$
p_{l} g\left(\tilde{\varsigma}_{l}\right)+p_{k} g\left(\tilde{\varsigma}_{k}\right) \leq p_{l} g\left(\varsigma_{l}\right)+p_{k} g\left(\varsigma_{k}\right)
$$

and, hence, $\sum_{j=1}^{n} p_{j} g\left(\tilde{\varsigma}_{j}\right) \leq \sum_{j=1}^{n} p_{j} g\left(\varsigma_{j}\right)$. Repeatedly applying this procedure results in a non-decreasing sequence $\tilde{\boldsymbol{\varsigma}}$ that satisfies $\boldsymbol{\sigma} \succeq_{p} \tilde{\boldsymbol{\varsigma}}$. But any such sequence is $p$-majorized by $\boldsymbol{\sigma}^{+}$. Hence, the statement of the lemma follows from Lemma 1.

Proof of Proposition 4. The statement of the proposition follows from a more general result established in Proposition 6 that also incorporates transfers into the support function.

Proof of Proposition 5. Using Proposition 4 and the definition of the interim support function we have

$$
\mathcal{S}^{D I C}(\boldsymbol{\Omega})=\mathcal{S}_{\text {interim }}\left(\boldsymbol{\Omega}^{+}\right)=\max \left\{\sum_{k \in \mathcal{K}} E_{\mathbf{x}}\left(q^{k}(\mathbf{x}) \sum_{i \in \mathcal{I}} a_{i}^{k} \Omega_{i}^{+}\left(x_{i}\right)\right) \mid \mathbf{q} \text { is feasible }\right\} .
$$

This establishes the optimality of the allocation rule in equation (8). To derive the payments that are ex post individually rational and implement allocation (8) in dominant strategies we consider constraints (3). Considering the payments binding the upward incentive constraints and the ex post individually rationality constraint for the lowest type we recursively calculate

$$
t_{i}(\mathbf{x})=v_{i}(\mathbf{x})-\sum_{x_{i}^{j}<x_{i}}\left(x_{i}^{j+1}-x_{i}^{j}\right) v_{i}\left(x_{i}^{j}, \mathbf{x}_{-i}\right)
$$

for $\mathbf{x} \in X$ and $i \in \mathcal{I}$. This establishes the claim of the proposition.

Proof of Proposition 6. Let us first derive the support function of feasible interim expected values and transfers that also satisfy Bayesian incentive compatibility (BIC) constraints (4) and interim individual rationality (INIR) constraints. For convenience we rewrite these constraints as

$$
\begin{aligned}
T_{i}\left(x_{i}^{j}\right)-T_{i}\left(x_{i}^{j-1}\right) & \geq x_{i}^{j-1}\left(V_{i}\left(x_{i}^{j}\right)-V_{i}\left(x_{i}^{j-1}\right)\right) \\
T_{i}\left(x_{i}^{j}\right)-T_{i}\left(x_{i}^{j-1}\right) & \leq x_{i}^{j}\left(V_{i}\left(x_{i}^{j}\right)-V_{i}\left(x_{i}^{j-1}\right)\right) \\
T_{i}\left(x_{i}^{j}\right) & \leq x_{i}^{j} V_{i}\left(x_{i}^{j}\right)
\end{aligned}
$$


The support function of the intersection of non-empty closed convex sets is the convolution of the support functions of these sets (see Rockafelar, 1970). When some sets are half spaces the support function of the intersection can be calculated using formula (5) and, hence, we obtain

$$
\mathcal{S}^{B I C}(\mathbf{W}, \mathbf{Z})=\inf _{\Lambda, \gamma, \mu \geq 0} E_{\mathbf{x}}\left(\max _{k \in \mathcal{K}} \sum_{i} a_{i}^{k} \widehat{W}_{i}\left(x_{i}\right)\right)+E_{\mathbf{x}}\left(\delta\left(\widehat{Z}_{i}\left(x_{i}\right)=0, \forall x_{i}, \forall i\right)\right)
$$

where

$$
\begin{aligned}
\widehat{W}_{i}\left(x_{i}^{j}\right) & =W_{i}\left(x_{i}^{j}\right)+\frac{1}{f_{i}\left(x_{i}^{j}\right)}\left(-x_{i}^{j-1} \Lambda_{i}\left(x_{i}^{j-1}\right)+x_{i}^{j} \Lambda_{i}\left(x_{i}^{j}\right)+x_{i}^{j} \gamma_{i}\left(x_{i}^{j}\right)-x_{i}^{j+1} \gamma_{i}\left(x_{i}^{j+1}\right)+x_{i}^{j} \mu_{i}\left(x_{i}^{j}\right)\right) \\
\widehat{Z}_{i}\left(x_{i}^{j}\right) & =Z_{i}\left(x_{i}^{j}\right)+\frac{1}{f_{i}\left(x_{i}^{j}\right)}\left(\Lambda_{i}\left(x_{i}^{j-1}\right)-\Lambda_{i}\left(x_{i}^{j}\right)-\gamma_{i}\left(x_{i}^{j}\right)+\gamma_{i}\left(x_{i}^{j+1}\right)-\mu_{i}\left(x_{i}^{j}\right)\right)
\end{aligned}
$$

and parameters $\Lambda_{i}\left(x_{i}^{j-1}\right), \gamma_{i}\left(x_{i}^{j}\right), \mu_{i}\left(x_{i}^{j}\right)$ correspond to constraints A.2, A.3, and A.4 respectively. Note that our convention is that $\Lambda_{i}\left(x_{i}^{0}\right)=\Lambda_{i}\left(x_{i}^{N_{i}}\right)=0$ and $\gamma_{i}\left(x_{i}^{1}\right)=\gamma_{i}\left(x_{i}^{N_{i}+1}\right)=0$. Since agents' utilities satisfy the single crossing condition with respect to $x_{i}$ and $V_{i}\left(x_{i}\right)$ the INIR constraints are binding only for the lowest type, i.e. $\mu_{i}\left(x_{i}^{j}\right)=0$ for $j=2, \ldots, N_{i}$. Using this fact and that $\left(\widehat{Z}_{i}\left(x_{i}\right)=0, \forall x_{i}, \forall i\right)$ we deduce

$$
\begin{aligned}
\mu_{i}\left(x_{i}^{1}\right) & =\sum_{l=1}^{N_{i}} Z_{i}\left(x_{i}^{l}\right) f_{i}\left(x_{i}^{l}\right) \\
\gamma_{i}\left(x_{i}^{j}\right) & =\sum_{l=j}^{N_{i}} Z_{i}\left(x_{i}^{l}\right) f_{i}\left(x_{i}^{l}\right)+\Lambda_{i}\left(x_{i}^{j-1}\right) \text { for } j=2, \ldots, N_{i}
\end{aligned}
$$

Note that constraints $\mu_{i}\left(x_{i}^{1}\right) \geq 0$ and $\gamma_{i}\left(x_{i}^{j}\right) \geq 0$ are satisfied for any vector $\mathbf{Z} \in \mathbb{R}_{+}^{\sum_{i}\left|X_{i}\right|}$. With some abuse of notation we replace $\left(x_{i}^{j+1}-x_{i}^{j}\right) \Lambda_{i}\left(x_{i}^{j}\right)$ with $\Lambda_{i}\left(x_{i}^{j}\right)$. Substituting the above expressions in the formula A.5 the support function for the set of feasible interim expected values and transfers that satisfy BIC and INIR constraints reduces to

$$
\mathcal{S}_{\text {interim }}^{B I C}(\mathbf{W}, \mathbf{Z})=\inf _{0 \leq \Lambda_{i}\left(x_{i}\right)} E_{\mathbf{x}}\left(\max _{k \in \mathcal{K}} \sum_{i \in \mathcal{I}} a_{i}^{k}\left(W_{i}\left(x_{i}\right)+M R_{Z_{i}}\left(x_{i}\right)-\frac{\Delta \Lambda_{i}\left(x_{i}\right)}{f_{i}\left(x_{i}\right)}\right)\right)
$$

Let us now define shifted weights

$$
\widehat{W}_{i}\left(x_{i}\right)=W_{i}\left(x_{i}\right)+M R_{Z_{i}}\left(x_{i}\right)-\frac{\Delta \Lambda_{i}\left(x_{i}\right)}{f_{i}\left(x_{i}\right)}
$$

It is straightforward to verify that $\mathbf{W}_{\mathbf{i}}+\mathbf{M R}_{\mathbf{Z}_{\mathbf{i}}} \succeq_{f_{i}} \widehat{\mathbf{W}}_{i}$ for all $i \in \mathcal{I}$. ${ }^{23}$ Therefore, Lemma A1 implies that $\left(\mathbf{W}_{\mathbf{i}}+\mathbf{M R}_{\mathbf{Z}_{\mathbf{i}}}\right)^{+}$delivers the minimum to the above expression, which establishes the claim of the proposition for the BIC support function.

${ }^{23}$ Note that $\sum_{j=1}^{l} \Delta \Lambda_{i}\left(x_{i}^{j}\right)=\Lambda_{i}\left(x_{i}^{l}\right)-\Lambda_{i}\left(x_{i}^{0}\right) \geq 0$ for $l=1, \ldots, N_{i}$ with equality for $l=N_{i}$. 
We now show that the introduction of the dominant strategy incentive compatibility (DIC) constraints

$$
\begin{aligned}
& t_{i}\left(x_{i}^{j}, \mathbf{x}_{-i}\right)-t_{i}\left(x_{i}^{j-1}, \mathbf{x}_{-i}\right) \geq x_{i}^{j-1}\left(v_{i}\left(x_{i}^{j}, \mathbf{x}_{-i}\right)-v_{i}\left(x_{i}^{j-1}, \mathbf{x}_{-i}\right)\right) \\
& t_{i}\left(x_{i}^{j}, \mathbf{x}_{-i}\right)-t_{i}\left(x_{i}^{j-1}, \mathbf{x}_{-i}\right) \leq x_{i}^{j}\left(v_{i}\left(x_{i}^{j}, \mathbf{x}_{-i}\right)-v_{i}\left(x_{i}^{j-1}, \mathbf{x}_{-i}\right)\right)
\end{aligned}
$$

and ex post individual rationality (EXIR) constraints

$$
t_{i}\left(x_{i}^{j}, \mathbf{x}_{-i}\right) \leq x_{i}^{j} v_{i}\left(x_{i}^{j}, \mathbf{x}_{-i}\right)
$$

lead to the same support function as the introduction of BIC and INIR constraints. To accomplish this we use the geometric interpretation of incentive constraints: the support function minimization problem corresponds to the intersection of the feasible set with the corresponding incentive constraint. Hence, we can include the constraints to support function (11) for one agent at a time.

Using arguments similar to the BIC support function we first include agent 1's DIC and EXIR constraints to the support function. Therefore,

$$
\begin{aligned}
\mathcal{S}_{\text {agent }_{1}}^{\text {DIC }}(\mathbf{W}, \mathbf{Z})=\inf _{0 \leq \lambda_{1}(\mathbf{x})} E_{\mathbf{x}}\left(\operatorname { m a x } _ { k \in \mathcal { K } } \left(a_{1}^{k}\left(W_{1}\left(x_{1}\right)+M R_{Z_{1}}\left(x_{1}\right)-\frac{\Delta \lambda_{1}(\mathbf{x})}{f_{1}\left(x_{1}\right)}\right)+\right.\right. \\
\left.\sum_{i \neq 1} a_{i}^{k}\left(W_{i}\left(x_{i}\right)+M R_{Z_{i}}\left(x_{i}\right)\right)\right)
\end{aligned}
$$

where parameters $\lambda_{1}\left(x_{1}^{j-1}, \mathbf{x}_{-1}\right)$ correspond to constraints A.6., $\lambda_{1}\left(x_{1}^{0}, \mathbf{x}_{-1}\right)=\lambda_{1}\left(x_{1}^{N_{1}}, \mathbf{x}_{-1}\right)=$ 0 , and $\Delta \lambda_{1}\left(x_{1}^{j}, \mathbf{x}_{-1}\right)=\lambda_{1}\left(x_{1}^{j}, \mathbf{x}_{-1}\right)-\lambda_{1}\left(x_{1}^{j-1}, \mathbf{x}_{-1}\right)$. We again consider the shifted weights

$$
\widehat{W}_{1}(\mathbf{x})=W_{1}\left(x_{1}\right)+M R_{Z_{1}}\left(x_{1}\right)-\frac{\Delta \lambda_{1}(\mathbf{x})}{f_{1}\left(x_{1}\right)}
$$

For each $\mathbf{x}_{-1}$ vector $\widehat{\mathbf{W}}_{1}\left(\cdot, \mathbf{x}_{-1}\right)$ satisfies $\mathbf{W}_{\mathbf{1}}+\mathbf{M R}_{\mathbf{Z}_{1}} \succeq_{f_{1}} \widehat{\mathbf{W}}_{1}\left(\cdot, \mathbf{x}_{-1}\right)$ and the above minimization problem can be rewritten as

$$
\sum_{\mathbf{x}_{-1}} \inf _{\mathbf{W}_{\mathbf{1}}+\mathbf{M R}_{\mathbf{Z}_{\mathbf{1}}} \succeq_{f_{1}} \widehat{\mathbf{w}}_{1\left(\cdot, \mathbf{x}_{-1}\right)}} \sum_{x_{1}} f_{1}\left(x_{1}\right) g_{1}\left(\widehat{W}_{1}\left(x_{1}, \mathbf{x}_{-1}\right)\right)
$$

where $g_{1}(y)=f_{-1}\left(\mathbf{x}_{-1}\right) \max _{k \in \mathcal{K}}\left(a_{1}^{k} y+\sum_{j \neq 1} a_{j}^{k}\left(W_{j}\left(x_{j}\right)+M R_{Z_{j}}\left(x_{j}\right)\right)\right.$ is a convex function of $y$. Lemma A1 asserts that $\widehat{\mathbf{W}}_{1}\left(\cdot, \mathbf{x}_{-1}\right)=\left(\mathbf{W}_{\mathbf{1}}+\mathbf{M R}_{\mathbf{Z}_{1}}\right)^{+}$for each $\mathbf{x}_{-1}$ solves the above minimization problem.

Let us now assume that we have introduced the constraints of $i-1$ agents. The minimization problem that corresponds to the introduction of the constraints of agent $i$ is

$$
\sum_{\mathbf{x}_{-i}} \inf _{\mathbf{W}_{\mathbf{i}}+\mathbf{M R}_{\mathbf{Z}_{\mathbf{i}}} \succeq_{i}} \sum_{\widehat{\mathbf{W}}_{i}\left(\cdot, \mathbf{x}_{-i}\right)} f_{x_{i}}\left(x_{i}\right) g_{i}\left(\widehat{W}_{i}\left(x_{i}, \mathbf{x}_{-i}\right)\right)
$$


where

$$
\widehat{W}_{i}(\mathbf{x})=W_{i}\left(x_{i}\right)+M R_{Z_{i}}\left(x_{i}\right)-\frac{\Delta \lambda_{i}(\mathbf{x})}{f_{i}\left(x_{i}\right)}
$$

and function

$$
g_{i}(y)=f_{-i}\left(\mathbf{x}_{-i}\right) \max _{k \in \mathcal{K}}\left(\sum_{j<i} a_{j}^{k}\left(W_{j}\left(x_{j}\right)+M R_{Z_{j}}\left(x_{j}\right)\right)^{+}+a_{i}^{k} y+\sum_{j>i} a_{j}^{k}\left(W_{j}\left(x_{j}\right)+M R_{Z_{j}}\left(x_{j}\right)\right)\right)
$$

is a convex function of $y$. Lemma $\mathrm{A} 1$ again asserts that $\widehat{\mathbf{W}}_{i}\left(\cdot, \mathbf{x}_{-i}\right)=\left(\mathbf{W}_{\mathbf{i}}+\mathbf{M R}_{\mathbf{Z}_{\mathbf{i}}}\right)^{+}$for each $\mathbf{x}_{-i}$ solves the above minimization problem. Proceeding in this way for all agents, we finally obtain that the support function for the feasible set of agent interim expected values and transfers that satisfies the DIC and EXIR constraints coincides with $\mathcal{S}^{B I C}(\mathbf{W}, \mathbf{Z})$.

Proof of Proposition 7. Vector $\left(\mathbf{V}^{*}, \mathbf{T}^{*}\right)$ belongs to $\nabla \mathcal{S}^{B I C}\left(\nabla \mathcal{O}\left(\mathbf{V}^{*}, \mathbf{T}^{*}\right)\right)$ if and only if (see Theorem 23.5 in Rockafellar, 1970)

$$
\left(\mathbf{V}^{*}, \mathbf{T}^{*}\right) \in \operatorname{argmax}\left((\mathbf{V}, \mathbf{T}) \circ \nabla \mathcal{O}\left(\mathbf{V}^{*}, \mathbf{T}^{*}\right) \mid(\mathbf{V}, \mathbf{T}) \in C\right)
$$

where $C$ is the set of feasible incentive compatible and individually rational interim expected values and transfers. This is equivalent to $\nabla \mathcal{O}\left(\mathbf{V}^{*}, \mathbf{T}^{*}\right)$ be tangent to set $C$ at $\left(\mathbf{V}^{*}, \mathbf{T}^{*}\right)$ (see p. 15, Rockafellar, 1997). Finally, Theorem 27.4 in Rochafellar (1997) establishes that this is equivalent to $\left(\mathbf{V}^{*}, \mathbf{T}^{*}\right)$ be a vector where maximum of $\mathcal{O}(\mathbf{V}, \mathbf{T})$ relative to $C$ is attained.

Proof of Proposition 8. We show in Subsection 3.1 that the support function for allocation probabilities $q^{k}(\mathbf{x})$ equals

$$
\mathcal{S}(\mathbf{w})=\sum_{x \in X} \max _{k \in \mathcal{K}} w^{k}(\mathbf{x})
$$

Taking into account how the support function changes under a linear transformation (see Subsection 3.1 we derive the support function for ex post agent values $v_{i}(\mathbf{x})=\sum_{k} v_{i}^{k}(\mathbf{x}) q^{k}(\mathbf{x})$ :

$$
\mathcal{S}_{\text {ex post }}(\mathbf{w})=\sum_{\mathbf{x} \in X} \max _{k \in \mathcal{K}} \sum_{i \in \mathcal{I}} v_{i}^{k}(\mathbf{x}) w_{i}(\mathbf{x})
$$

The interim expected values are given by $V_{i}\left(\mathbf{x}_{i}\right)=\sum_{\mathbf{x}_{-i}} f\left(\mathbf{x} \mid \mathbf{x}_{i}\right) v_{i}(\mathbf{x})$, where $f\left(\mathbf{x} \mid \mathbf{x}_{i}\right)$ denotes the conditional distribution of agent types. Therefore,

$$
\mathcal{S}_{\text {interim }}(\mathbf{W})=E_{\mathbf{x}}\left(\max _{k \in \mathcal{K}} \sum_{i \in \mathcal{I}} v_{i}^{k}(\mathbf{x}) W_{i}\left(x_{i}\right)\right)
$$

where, as before, we multiplied by $f_{i}\left(\mathbf{x}_{i}\right)$, i.e. we used the probability weighted inner product to define the set of interim expected values. 
Proof of Proposition 9. The statement for BIC mechanisms directly follows from Theorem 3.1 in Jehiel and Moldovanu (2001). The extension to EPIC mechanisms is immediate.

Proof of Proposition 10. The second-best level of social surplus equals the value of support function at unit weights $S^{B I C}(\mathbf{1})$. If the auctioneer has to always allocate the unit we have

$$
S^{B I C}(\mathbf{1})=\alpha\left(E_{i}\left(x_{i}\right)+E_{j}\left(x_{j}\right)\right)+\inf _{\Lambda_{i} \geq 0} E_{\mathbf{x}} \max _{i \neq j}\left((1-\alpha) x_{i}-\frac{\Delta \Lambda_{i}\left(x_{i}\right)}{f_{i}\left(x_{i}\right)}\right)
$$

If we denote the shifted weights $\widehat{W}_{i}\left(x_{i}\right)=(1-\alpha) x_{i}-\frac{\Delta \Lambda_{i}\left(x_{i}\right)}{f_{i}\left(x_{i}\right)}$ Lemma A1 establishes that $\mathrm{f}$ majorized values $\left((1-\alpha) x_{i}\right)^{+}=(1-\alpha) E_{i}\left(x_{i}\right)$ deliver the minimum to the above expression. After some rearrangement the second-best level of social surplus equals

$$
S^{B I C}(\mathbf{1})=\max _{i} E_{i}\left(x_{i}\right)+\alpha \min _{i} E_{i}\left(x_{i}\right)
$$

The expression of $S^{E P I C}(\mathbf{1})$ can be written similarly. The only differences is that the minimization takes place over $\lambda_{i}(\mathbf{x}) \geq 0$ that depend on the whole vector of agent types. The argument of Proposition 6 establishes that $\left((1-\alpha) x_{i}\right)^{+}$delivers also the minimum to support function $S^{E P I C}(\mathbf{1})$ with the same optimal value. Hence, the second-best allocation can be implemented with some EPIC and EXIR mechanism mechanism.

Proof of Proposition 11. If the auctioneer can keep the unit the value of support function at unit weights equals

$$
S^{B I C}(\mathbf{1})=\alpha\left(E_{i}\left(x_{i}\right)+E_{j}\left(x_{j}\right)\right)+\inf _{\Lambda_{i} \geq 0} E_{\mathbf{x}} \max _{i \neq j}\left(-\alpha\left(x_{i}+x_{j}\right),\left((1-\alpha) x_{i}-\frac{\Delta \Lambda_{i}\left(x_{i}\right)}{f_{i}\left(x_{i}\right)}\right)\right.
$$

Given the condition on $\alpha$ stated in the proposition and that agent types are positive we have

$$
\left((1-\alpha) x_{i}\right)^{+}=(1-\alpha) E_{i}\left(x_{i}\right)>-\alpha\left(x_{i}+x_{j}\right)
$$

for each realization of $x_{i}$ and $x_{j}$ for at least some $i \neq j=1,2$. Hence, the shifted weights $\widehat{W}_{i}\left(x_{i}\right)=\left((1-\alpha) x_{i}\right)^{+}$identified in Proposition 10 still deliver the minimum to the minimization problem for $S^{B I C}(\mathbf{1})$. The same argument applies to $S^{D I C}(\mathbf{1})$. 


\section{References}

Alaei, Saeed, Hu Fu, Nima Haghpanah, Jason Hartline, and Azarakhsh Malekian (2012) "Bayesian Optimal Auctions via Multi- to Single-agent Reduction," Working Paper.

Aliprantis, Charalambos D. and Kim C. Border (2006) "Infinite Dimensional Analysis: A Hitchhiker's Guide," Springer, Berlin.

Armstrong, Mark. (2000) "Optimal Multi-Object Auctions," Review of Economic Studies, 67, $455-481$.

Asker, John and Estelle Cantillon (2010) "Procurement when price and quality matter," The RAND Journal of Economics, 41, 1-34.

Ayres, Ian and Peter Crampton (1996) "Deficit Reduction Through Diversity: How Affirmative Action at the FCC Increased Auction Competition," Stanford Law Review, 48, 761-815.

Baldwin, Elizabeth and Paul Klemperer (2012) "Tropical Geometry to Analyse Demand," Working paper, Oxford University.

Bapat, R. B. (1991) "Majorization and Singular Values III, " Linear Algebra and its Applications, 145, 59-70.

Belloni, Alexandre, Giuseppe Lopomo, and Shouqiang Wang (2010) "Multidimensional Mechanism Design: Finite-Dimensional Approximations and Efficient Computation," Operations Research, 58, 1079-1089.

Bergemann, D. and Morris, S. (2005): "Robust Mechanism Design," Econometrica, 73, 17711813.

Border, Kim C. (1991) "Implementation of Reduced Form Auctions: A Geometric Approach," Econometrica, 59, 1175-1187.

Border, Kim C. (2007) "Reduced Form Auctions Revisited," Economic Theory, 31, 167-181.

Brusco, Sandro and Giuseppe Lopomo (2002) "Collusion via Signalling in Simultaneous Ascending Bid Auctions with Heterogeneous Objects, with and without Complementarities," The Review of Economic Studies, 69, 407-436.

Cai, Yang, Constantinos Daskalakis, and Matt Weinberg (2012a) "An Algorithmic Characterization of Multi-Dimensional Mechanisms," in the 44th ACM Symposium on Theory of Computing.

Cai, Yang, Constantinos Daskalakis, and Matt Weinberg (2012b) "Optimal Multi-Dimensional Mechanism Desgin: Reducing Revenue to Welfare Maximization," in the 53rd Annual IEEE Symposium on Foundations of Computer Science. 
Che, Yeon-Koo, Daniele Condorelli, and Jinwoo Kim (2010) "Weak Cartels and Collusion-Proof Auctions," Working paper, Columbia University.

Che, Yeon-Koo, Jinwoo Kim, and Konrad Mierendorff (2011) "Generalized Reduced-Form Auctions: A Network-Flow Approach," Working paper, University of Zürich.

Fuchs, Ladislas (1947) "A New Proof of an Inequality of Hardy-Littlewood-Polya," Mat. Tidsskr. B, 53-54.

Gershkov, Alex, Jacob K. Goeree, Alexey Kushnir, Benny Moldovanu, and Xianwen Shi (2013) "On the Equivalence of Bayesian and Dominant-Strategy Implementation," Econometrica, 81, 197-220.

Goeree, Jacob K. and Alexey Kushnir (2013a) "Optimal Mechanisms for General Welfare Objectives," Working Paper, University of Zürich.

Goeree, Jacob K. and Alexey Kushnir (2013b) "A Geometric Approach to Mechanism Design: The Case of Multidimensional Types," Working Paper, University of Zürich.

Gutmann, Sam, J. H. B. Kemperman, J. A. Reeds, and L. A. Shepp (1991) "Existence of Probability Measures with Given Marginals," Annals of Probability, 19, 1781-1797.

Hardy, Godfrey H., Littlewood, John E., and Pólya, Gyorgy (1929) "Some simple inequalities satisfied by convex functions," Messenger Math, 58, 145-152.

Hart, Sergiu and Philip J. Reny (2011) "Implementation of Reduced Form Mechanisms: A Simple Approach and a New Characterization," Working Paper, The Hebrew University.

Hernando-Veciana, Angel and Fabio Michelucci (2009) "Second-Best Efficiency and the English Auction," Working Paper.

Hernando-Veciana, Angel and Fabio Michelucci (2011) "Second-Best Efficiency and the English Auction," Games and Economic Behavior, 73, 496-506.

Hörner, Johannes and Larry Samuelson (2011) "Managing Strategic Buyers," Journal of Political Economy, 119, 379-425.

Kushnir, Alexey (2013) "On the Equivalence of Bayesian and Dominant-Strategy Implementation: The Case of Correlated Types," Working Paper, University of Zürich.

Ledyard, John O. and Thomas R. Palfrey J. (1999) "A characterization of interim efficiency with public goods," Econometrica, 67, 435-848.

Ledyard, John O. and Thomas R. Palfrey (2007) "A general characterization of interim efficient mechanisms for independent linear environments," Journal of Economic Theory 133, $441-466$. 
Manelli, Alejandro M. and Daniel R. Vincent (207) "Multidimensional Mechanism Design: Revenue Maximization and the Multiple-good Monopoly," Journal of Economic Theory, 137, 153-185.

Manelli, Alejandro M. and Daniel R. Vincent (2010) "Bayesian and Dominant-Strategy Implementation in the Independent Private Values Model," Econometrica, 78, 1905-1938.

Marshall, A. W., I. Olkin, I. and B. C. Arnold (2011) Inequalities: Theory of Majorization and its Applications, Springer, New York.

Maskin, Eric (1992) "Auctions and Privatization," in Privatization: Symposium in honour of Herbert Giersh, ed. by H. Siebert, Institute fur Weltwirtshaft an der Univarsitat Kiel.

Maskin, Eric and John Riley (1984) "Optimal Auctions with Risk Averse Buyers," Econometrica, 52, 1473-1518.

Matthews, Steven A. (1984) "On the Implementability of Reduced Form Auctions," Econometrica, 52, 1519-1522.

Mierendorff, Konrad (2011) "Asymmetric Reduced Form Auctions," Economics Letters, 110, 41-44.

Mierendorff, Konrad (2011) “Optimal Dynamic Mechanism Design with Deadlines," Working Paper, University of Zürich.

Milgrom, Paul and Ilya Segal (2002) "Envelope Theorems for Arbitrary Choice Sets," Econometrica, 70, 583-601.

Miralles, Antonio (2012) "Cardinal Bayesian Allocation Mechanisms Without Transfers," Journal of Economic Theory, 147, 179-206.

Mussa, Michael and Sherwin Rosen (1978) "Monopoly and Product Quality," Journal of Economic Theory, 18, 301-317.

Myerson, Roger B. (1981) "Optimal Auction Design," Mathematics of Operations Research, 6, $58-73$.

Magill, Michael, Martine Quinzii and Jean-Charles Rochet (2010)"Reforming Capitalism," Working Paper.

Pai, Mallesh (2009) "Competing Auctioneers," Working Paper, Kellogg School of Management, Northwestern University.

Pai, Mallesh and Rakesh Vohra (2010) "Optimal Auctions with Financially Constrained Bidders," Working Paper, Kellogg School of Management, Northwestern University.

Pai, Mallesh and Rakesh Vohra (2011) "Otimal Dynamic Auctions and Simple Index Rules," 
Working Paper, Kellogg School of Management, Northwestern University.

Pai, Mallesh and Rakesh Vohra (2012) "Auction Design with Fairness Concerns: Subsidies vs. Set-Asides," Working Paper, Kellogg School of Management, Northwestern University.

Polishchuk, Leonid, and Alexander Tonis (2013) "Endogenous contest success functions: a mechanism design approach," Economic Theory, 52, 271-297.

Rochet, Jean-Charles (1987) "A necessary and sufficient condition for rationalizability in a quasi-linear context," Journal of Mathematical Economics, 16, 191-200.

Rockafellar, R. Tyrrell (1970) Convex Analysis, Princeton Landmarks in Mathematics, Princeton University Press.

Tirole, Jean (2001) "Corporate Governance," Econometrica, 69,1-35.

Vohra, Rakesh V. (2011) Mechanism Design: A Linear Programming Approach, Cambridge University Press. 